\title{
The mechanism of internal fatigue-crack initiation and early growth in a titanium alloy with lamellar and equiaxed microstructure
}

\author{
Xiangnan Pan ${ }^{\mathrm{a}, \mathrm{b}}$, Shouwen Xu ${ }^{\mathrm{a}, \mathrm{b}}$, Guian Qian ${ }^{\mathrm{a}, \mathrm{b}}$, Alexander Nikitin ${ }^{\mathrm{c}, \mathrm{d}}$, Andrey Shanyavskiy ${ }^{\mathrm{e}}$, \\ Thierry Palin-Luc ${ }^{\mathrm{f}}$, Youshi Hong ${ }^{\mathrm{a}, \mathrm{b}, *}$ \\ ${ }^{a}$ LNM, Institute of Mechanics, Chinese Academy of Sciences, Beijing, 100190, China \\ ${ }^{\mathrm{b}}$ School of Engineering Science, University of Chinese Academy of Sciences, Beijing, 100049, China \\ ${ }^{\mathrm{c}}$ LEME, University Paris Ouest Nanterre La Defense, 50 rue de Serves, Ville-d'Avray, 92410, France \\ ${ }^{\mathrm{d}}$ MAI - National Research University, 4 Volokolamskoe Hwy, A-80, GSP-3, Moscow, 125993, Russia \\ ${ }^{\text {e }}$ Aviaregister, Air. Sheremetevo-1, PO Box 54, Moscow Reg, Chimkovskiy State, 141426, Russia \\ ${ }^{\mathrm{f}}$ Arts et Metiers Institute of Technology, CNRS, Universite de Bordeaux, Bordeaux INP, INRAE, I2M Bordeaux, Esplanade des Arts et Metiers, Talence, 33405, France
}

\section{A R T I C L E I N F O}

\section{Keywords:}

Titanium alloy

Crack initiation

Facet

Very-high-cycle fatigue

Lamellar microstructure

\begin{abstract}
A B S T R A C T
Traditionally, equiaxed $\alpha$ grains rather than lamellar microstructure (LM) domains in titanium alloys are regarded as potential internal crack origins in high-cycle fatigue (HCF) and very-high-cycle fatigue (VHCF) regimes. Here, we found that the fatigue crack is prone to initiate from a large LM domain in a titanium alloy with the composition of LM and equiaxed microstructure (EM) of fine $\alpha$ grains. Then, the mechanisms of internal crack initiation and early growth for the cases of HCF and VHCF under stress ratio $R=-1,0.1$ and 0.5 were addressed and a mechanism chart was constructed to illustrate the internal cracking behavior, especially showing that the numerous cyclic pressing process dominates the related microstructure evolution with grain size refinement and nanograin formation underneath the fracture surfaces in the region of crack initiation and early growth.
\end{abstract}

\section{Introduction}

Titanium alloys have many good properties, such as high strength, low mass density and excellent corrosion resistance, which make them be broadly used in a variety of engineering structures, like aircrafts, aero-engines, bio-medical devices, and components in chemical processing equipment [1-3]. In general, titanium alloys can be classified into four types in terms of the possessed microstructural phase: near $\alpha$, $\alpha+\beta$, metastable $\beta$ and $\beta$ titanium alloys. Near $\alpha$ and $\alpha+\beta$ types are widely used alloys, in which $\alpha$ and $\beta$ phases coexist in the matrix. The shapes (equiaxed or lamellar) and the distribution of grains construct four typical microstructures: equiaxed microstructure (EM), bimodal microstructure (BM), basketweave microstructure (BW) and lamellar microstructure (LM). For near $\alpha$ and $\alpha+\beta$ titanium alloys, equiaxed $\alpha$ (or primary $\alpha$ ), lamellar $\alpha$ (or secondary $\alpha$ ) and lamellar $\beta$ grains prevail, and the microstructure contains no equiaxed $\beta[1,2]$.

For most engineering structures, fatigue resistance is a key safety factor in actual service requirement, especially in high-cycle (between $10^{5}$ and $10^{7}$ cycles) and very-high-cycle (beyond $10^{7}$ cycles) regimes [4-6]. There is no traditional endurance limit in titanium alloys $[7,8]$, and the number of cycles to failure $N_{\mathrm{f}}$ may reach $10^{7}$ and even $10^{9}$, i.e. very-high-cycle fatigue (VHCF). Consequently, the VHCF behavior of titanium alloys has drawn much more attention in recent decades [9-13].

Crack initiation is always an important issue of fatigue resistance, which consumes the majority of total fatigue life $[14,15]$. In particular, this is more than $90 \%$ at $N_{\mathrm{f}}=10^{7}$ and more than $99 \%$ at $N_{\mathrm{f}}>10^{8}$ cycles for a titanium alloy [15]. For titanium alloys with EM or with BM, the crack initiation area on the fracture surface of failed specimens in high-cycle fatigue (HCF) and VHCF regimes is easy to be identified [7, 15-20]. However, for the titanium alloys with BW or LM, it is difficult to locate the initiation site of fatigue cracks under HCF and VHCF [17, 21-23].

There are no pre-existing defects, such as inclusions, cavities or voids in the titanium alloys manufactured by casting or forging processes [1, 2]. Neal and Blenkinsop [16] first reported that fatigue crack originated from specimen interior with fish-eye (FiE) morphology in two $\alpha+\beta$ type titanium alloys $\mathrm{Ti}-4 \mathrm{Al}-4 \mathrm{Mo}-2 \mathrm{Sn}-0.5 \mathrm{Si}$ and $\mathrm{Ti}-6 \mathrm{Al}-4 \mathrm{~V}$ under axial cycling with stress ratio $R=0$ in HCF and VHCF regimes. The fractographic observations showed facet features in crack initiation region with rough morphology called rough area (RA) almost at the center of

\footnotetext{
* Corresponding author. LNM, Institute of Mechanics, Chinese Academy of Sciences, Beijing, 100190, China.

E-mail address: hongys@imech.ac.cn (Y. Hong).
} 


\section{Nomenclature and abbreviations}

$\begin{array}{ll}\text { BM } & \text { Bimodal microstructure } \\ \text { BW } & \text { Basketweave microstructure } \\ \text { EM } & \text { Equiaxed microstructure } \\ \text { FIB } & \text { Focused ion beam } \\ \text { LM } & \text { Lamellar microstructure } \\ \text { HCF } & \text { High-cycle fatigue } \\ \text { NCP } & \text { Numerous cyclic pressing } \\ N_{\mathrm{f}} & \text { Number of cycles to failure } \\ R & \text { Stress ratio } \sigma_{\min } / \sigma_{\max } \\ \text { SAD } & \text { Selected area electron diffraction } \\ \text { SEM } & \text { Scanning electron microscopy } \\ \text { SWLI } & \text { Scanning white light interferometry } \\ \text { TEM } & \text { Transmission electron microscopy } \\ \text { VHCF } & \text { Very-high-cycle fatigue }\end{array}$

FiE region. The analyses indicated that the facets nucleated from equiaxed $\alpha$ grains in these cases of EM or BM. They also postulated a mechanism to ascribe the facet formation to the cleavage at the crystal planes $\{10 \overline{1} 7\}$ by considering the results of Wanhill [24].

In general, the facet or facet-like morphology may appear in the fracture origin of titanium alloys with various microstructures in both HCF and VHCF regimes under specific $R$ values with or without FiE/RA pattern [16-23]. For titanium alloys with EM, facets occurred only in the RA regions [17-20]; and for BW and LM types, facet-like surfaces irregularly distributed on the fractured specimens without FiE and RA morphology [17,21-23]. Unlike the microstructures of EM and BM, the BW and the LM possess almost no equiaxed $\alpha$ grains [1,2].

Eylon and Hall [21] called the facet-like surface as "cleavage-like facet" in a titanium alloy (Ti-6Al-5Zr-0.5Mo-0.34Si) with BW or LM. Then, Evans and Bache [25-27] used the term "quasi-cleavage facet" to represent this feature in the similar materials, and proposed a slip-based model to predict the facet-like fracture nucleation within a prior $\beta$ grain. In the titanium alloys with BW or LM, the prior $\beta$ grains are usually the LM domains with similar crystal orientations, which may fracture to form the facet-like surface in the manner of "quasi-cleavage". The model by Evans and Bache [25-27] are still suitable for the titanium alloys with $\mathrm{EM}$, in which the facet-like surfaces from the prior $\beta$ grains should be replaced by the facets within the equiaxed $\alpha$ grains. In this paper, we just used the terms "facet" and "quasi-facet" to describe different types of planar fracture surfaces.

Later on, several other mechanisms and results [28-34] described the facet formation in the equiaxed $\alpha$ grains or $\alpha$ lamella domains with similar orientations in the titanium alloys with homogeneous microstructures, such as EM, BW or LM. Nevertheless, the BM type titanium alloys are with the highest strength in HCF [35] and VHCF [19,22,23, 36] regimes, but the facet formation mechanism is still unclear and the related investigation is quite rare.

The BM is with both equiaxed $\alpha$ grains and LM domains [1,2]. It was observed that cracks are more prone to initiate from equiaxed $\alpha$ grains under cyclic loading [16,17]. Based on this point, Ravi Chandran [37] treated equiaxed (or primary) $\alpha$ grains as the potential defects and constructed a model of $2 \mathrm{D}$ Poisson distribution to predict the failure modes of surface or internal fatigue crack initiation containing facet morphology, and several results [19,36-39] were successively published to support this model.

Titanium alloys with EM have sufficient equiaxed $\alpha$ grains, and those with BM have not. Thus, surface crack initiation occurs in VHCF regime of those with EM $[18,19]$ and internal crack induced VHCF may happen in those with BM $[40,41]$. For the titanium alloys with EM or BM, the morphologies of FiE and RA are the typical fractographic features in HCF and VHCF [7,15-20,36-44], and the RA is a characteristic region of fracture process [15,18,19]. But for BW and LM types [17,21-23], there are no typical feature of FiE and RA on the fatigue fracture surface. Therefore, it is necessary to further investigate the propensity of crack initiation in different microstructures of titanium alloys in HCF and VHCF regimes.

It is also noted that no facet but fine granules are presented in the RA region of the titanium alloys with EM [18,19] and with BM [15,40,41] when the failures occur in HCF and VHCF regimes under fully reversed axial cycling $(R=-1)$. The mean normal stress, $\sigma_{\mathrm{m}}$, can significantly affect both the morphology and the position of RA. As $\sigma_{\mathrm{m}}$ increases, crack initiation site shifts from specimen surface to interior with the appearance of facets, and the facets are more evident in the RA region at high $R$ values [19,39,42-44]. Additionally, the microstructure evolution underneath the RA surface also differs under negative or positive stress ratios in VHCF regime. Nanograin layer was produced underneath the fracture surface within the RA region of a Ti-6Al-4V with EM $[18,19]$ and with BM [15] under $R=-1$, and the original coarse grains remained at $R=0$ and 0.5. Oguma and Nakamura [45] proposed a "cold-welding" model to describe the formation of the granular RA region in a titanium alloy. Hong et al. [46] proposed a new model of numerous cyclic pressing (NCP) to explain the formation process of fine granular area (FGA) in the crack initiation region in VHCF for high-strength steels. The NCP model has been validated not only for high-strength steels [46-49] but also for the RA of titanium alloys $[15,18,19]$. The importance of crack closure effects and repeated contacts due to compressive stress is emphasized in both models $[10,45,46]$. However, how the process of crack closure and surface contact influences the crack initiation and propagation especially for the titanium alloy with EM and LM still remains unclear.

In this paper, a titanium alloy VT3-1 (Ti-6Al-2Mo-1.25Cr) with both EM and LM was used to investigate the internal crack initiation mechanism in HCF and VHCF regimes. This titanium alloy has an EM with fine equiaxed $\alpha$ grains and an LM with coarse domains of transformed $\beta$ colonies. Four specimens with internal crack initiation, which were failed in HCF and VHCF regimes under negative and positive stress ratios, were selected for the examinations by means of fracture surface topography via scanning electron microscopy (SEM) and scanning white light interferometry (SWLI). Furthermore, microstructure characterizations underneath the fractographic features were conducted by focused ion beam (FIB) technique in combination with SEM and transmission electron microscopy (TEM) examinations. Based on the experimental results, a mechanism chart was sketched to illustrate the internal crack initiation and early growth under different stress ratios for this $\alpha+\beta$ titanium alloy in HCF and VHCF regimes.

\section{Material and methods}

\subsection{Tested titanium alloy with $L M$ and $E M$}

A VT3-1 titanium alloy (Russian code) was used in the present investigation. Its chemical composition (weight percentage) is: $6.3 \mathrm{Al}$, 2.0 Mo, 1.25 Cr, $0.46 \mathrm{Fe}, 0.32 \mathrm{Si}$ and balance Ti. This alloy is an $\alpha+\beta$ type titanium alloy, which was processed by extrusion and thus contained both LM and EM microstructures [50-53].

Fig. 1a-c presents the microstructure perpendicular to the extruded direction observed by SEM (with a JEOL JSM IT-300). The metallographic sample was carefully ground, polished down to 2000 grit silicon carbide paper, and etched with Kroll's reagent (1-3\% $\mathrm{HF}, 2-6 \% \mathrm{HNO}_{3}$ ). The cross sections of planes d-g marked by the red bars in Fig. $1 \mathrm{~b}$ and $\mathrm{c}$ were milled and imaged by a FIB/SEM dual-beam system with a FEI Helios NanoLab 460HP, and the observations are shown in Fig. 1d-g.

The lamellar and equiaxed microstructures were identified by the three micrographs, respectively: Fig. $1 \mathrm{~b}, \mathrm{~d}$ and e for LM, and Fig. 1c, $\mathrm{f}$ and $g$ for EM. It is seen that the feature of LM is of three levels: (1) isolated LM plates (a couple of $\alpha$ or $\beta$ layers), (2) LM colony (domain) with a few $\alpha$ and $\beta$ lamellae, which is originated from one prior $\beta$ grain, 

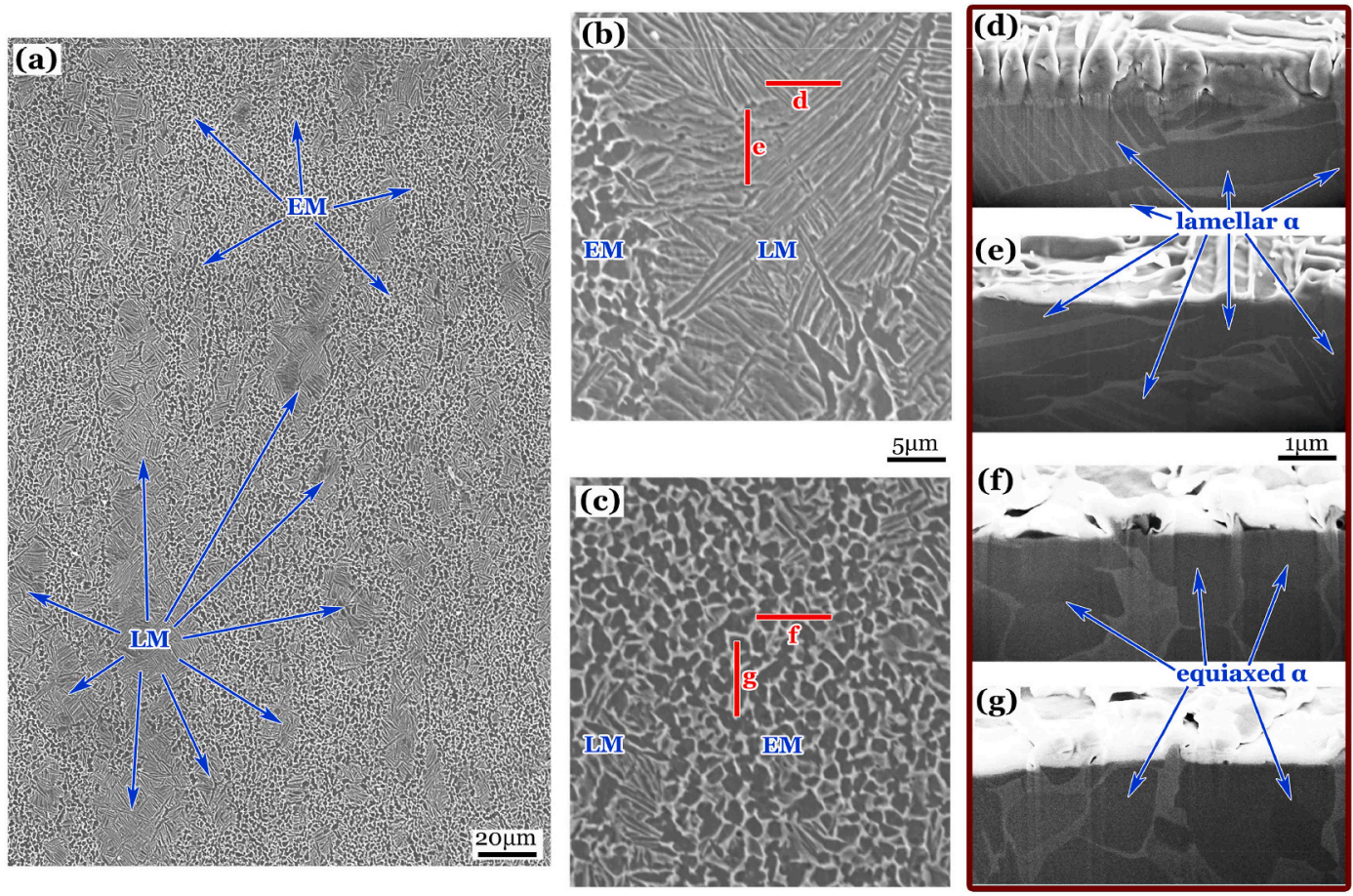

Fig. 1. Microstructure characterization perpendicular to the extruded direction, (a) medium magnification of SEM image, (b, c) high magnification of SEM images, and $(\mathrm{d}-\mathrm{g})$ micrographs of FIB-SEM images from the section planes $\mathrm{d}-\mathrm{g}$ marked by the red bars in (b, c). EM: equiaxed microstructure, LM: lamellar microstructure. (For interpretation of the references to color in this figure legend, the reader is referred to the Web version of this article.)
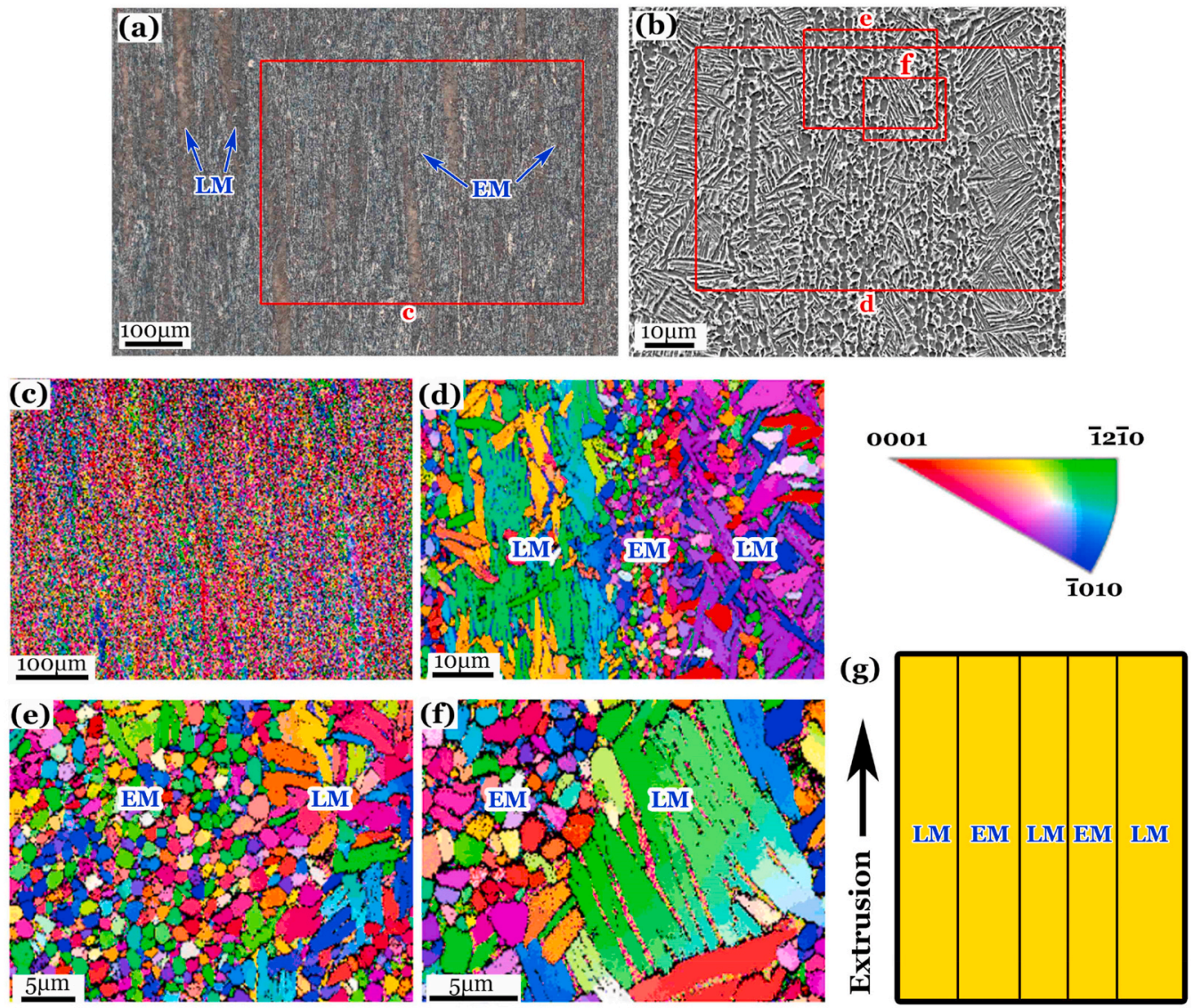

Fig. 2. Microstructure characterization along the extruded direction. (a) Low magnification OM image, (b) medium magnification SEM image, (c-f) IPFs of EBSD for the boxes c-f in (a) and (b), and (g) schematic sketch showing the distribution of LM and EM along the extruded direction. EM: equiaxed microstructure, LM: lamellar microstructure. 
and (3) LM cluster containing several colonies.

A lamellar colony or domain in the VT3-1 titanium alloy is a bundle of plate-like microstructure, and a group of lamellar domains (colonies) compose an LM column which is along the extrusion direction. For each lamellar plate, the ratio of length or width to thickness is between 10 and 20. The shape and the size of LM plates and colonies are of remarkable scattering: (1) the thickness of a lamellar plate is from submicron to $2 \mu \mathrm{m}$ and the length or width is from 3 to $20 \mu \mathrm{m}$, and (2) LM colonies are usually of trapezoid shape with the size (mean free path) between 3 and $20 \mu \mathrm{m}$. The LM colonies are randomly distributed in the matrix of EM whose average size is about $1-2 \mu \mathrm{m}$. Fig. 2 presents the microstructure along the extruded direction. Fig. 2a is a low magnification image of optical microscopy (OM) via a Zeiss Axiovert 200 MAT, Fig. $2 \mathrm{~b}$ is a medium magnification image by using the same SEM as mentioned in Fig. 1, and Fig. 2c-f are inverse pole figures (IPFs) of electron backscatter diffraction (EBSD) obtained via a field emission scanning electron microscope (FE-SEM) of Zeiss Merlin equipped with a NordlysNano detector (acceleration voltage: $20 \mathrm{kV}$ and scanning step: $100 \mathrm{~nm}$ ). The EBSD samples were first ground with silicon carbide papers down to 4000 grit, then polished with a $9 \mu \mathrm{m}$ diamond suspension, and finally polished with a 9:1 solution (colloidal silica suspension with a mean particle size of $0.04 \mu \mathrm{m}$ versus $\mathrm{H}_{2} \mathrm{O}_{2}$ ).

The microstructure in the longitudinal section paralleled to the extruded direction has typical texture features. Most of LM clusters are neatly arranged to form the rows of LM columns and some equiaxed $\alpha$ grains are elongated in the extrusion direction. In the gap region between two LM columns, the majority contents are equiaxed grains although such a region may contain a small amount of elongated $\alpha$ grains and small size LM domains. The EBSD results shown in Fig. 2c-f indicate that all $\alpha$ lamellae within an LM colony have similar crystal orientations, each LM colony within an LM cluster has its own orientation, there are always large LM clusters (tens of microns in size) with similar orientations within each LM column, and all equiaxed and elongated $\alpha$ grains are randomly distributed with different crystal orientations.

Here, the microstructure of the tested material with LM and EM is schematically illustrated in Fig. 2g. An idealized construction of fiber/ matrix composite is then sketched, where the LM columns can be considered as the coarse fibers and the EM can be regarded as the fine matrix. The spacing and the size of LM columns are tens of micrometers.

\subsection{Tensile and fatigue properties of the tested material}

The tensile properties and fatigue strength in the extruded direction of the tested titanium alloy were previously obtained [50-53]. The tensile specimen is of a flat shape with the gauge length of $25 \mathrm{~mm}$ and the thickness of $1 \mathrm{~mm}$. The tensile tests were conducted with a displacement rate of $0.075 \mathrm{~mm} / \mathrm{min}$. The tensile properties are: elastic modulus $E=106 \mathrm{GPa}$, dynamic elastic modulus $E_{\mathrm{d}}=110 \mathrm{GPa}$, ultimate tensile strength $\sigma_{\mathrm{u}}=1107 \mathrm{MPa}$, yield strength (with $0.2 \%$ plastic strain) $\sigma_{0.2}=1050 \mathrm{MPa}$ and elongation at rupture $\delta=13 \%$. The fatigue properties under different stress ratios of $R=-1,0.1$ and 0.5 are shown in Fig. 3. They were obtained by ultrasonic fatigue testing method [6] with the loading frequency of $20 \mathrm{kHz}$ at room temperature and in air. The fatigue specimen is of an hourglass shape with the reduced section diameter of $3.0 \mathrm{~mm}$ and the total length of $67.6 \mathrm{~mm}$. Details about both the specimen geometry and the testing procedure can be found in Refs. [50-53].

All the failed specimens in Fig. 3 exhibit the characteristics of internal crack initiation. For $R=-1$, the range of $N_{\mathrm{f}}$ is between $4.77 \times 10^{6}$ and $1.31 \times 10^{9}$ cycles, and the fatigue resistance in HCF and VHCF regimes has the same value of the maximum stress $\sigma_{\max }=400 \mathrm{MPa}$. For $R$ $=0.1$, the range of $N_{\mathrm{f}}$ is between $1.14 \times 10^{7}$ and $7.39 \times 10^{8}$ cycles, and the VHCF resistance almost keeps constant of $\sigma_{\max }=680 \mathrm{MPa}$. For $R=$ 0.5 , the VHCF resistance also keeps constant of $\sigma_{\max }=960 \mathrm{MPa}$. Based on this result, the failed specimens are classified into four loading

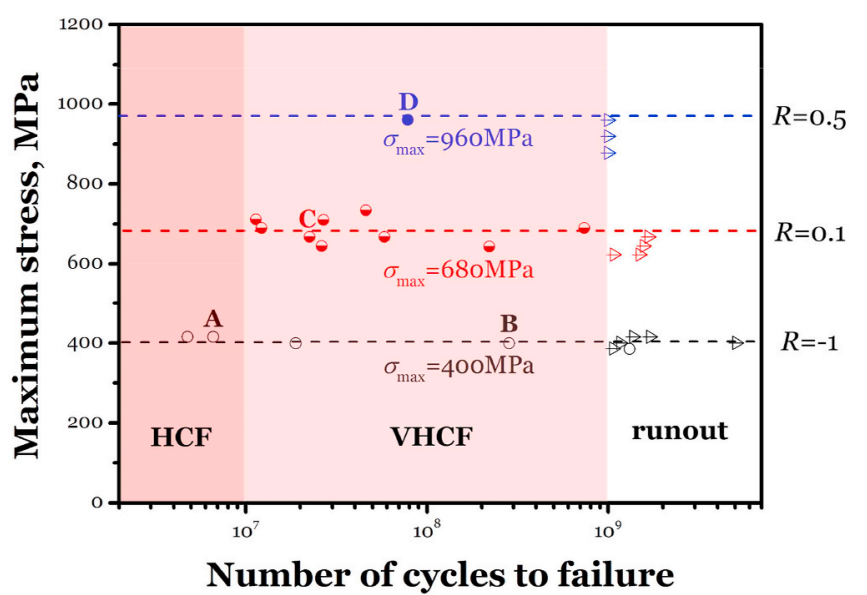

Fig. 3. S-N data of the tested VT3-1 extruded titanium alloy under different stress ratios of $R=-1,0.1$ and 0.5 [50-53]. (Symbols with a horizontal bar being run-out specimens).

conditions: (1) $R=-1$, HCF; (2) $R=-1$, VHCF; (3) $R=0.1$, VHCF; and (4) $R=0.5$, VHCF.

\subsection{Characterization methods for fracture surfaces}

Four fatigue failed specimens (labeled A, B, C and D in Fig. 3) were selected with regard to the four loading conditions for a detailed examination of the fractography and microstructure features underneath the fracture surfaces, especially in the crack initiation regions. The fatigue data of the four selected specimens are listed in Table 1.

The SEM samples for fractography observation were cut from one fracture end of each failed specimen of A, B, C and D with the sample height of about $7 \mathrm{~mm}$, which is suitable for sitting in the SEM chamber for observation. The samples were carefully observed by using SEM (JEOL JSM IT-300) with a CCD (charge coupled device) camera to obtain the large field fractographs of image resolution at $5120 \times 4096$ pixels under low and medium magnifications. The roughness of fracture surfaces was measured by means of topography analysis on the selected locations on the fracture surfaces of specimens A-D by using a noncontact optical 3D profiler (ZYGO Nexview) in accordance to ISO/DIS 25178-604 Part D. The profiler uses SWLI (scanning white light interferometry) technique to image and examine the microstructure, and the surface topography is of a resolution of $0.1 \mathrm{~nm}$ on $z$ (height) direction, where the focal plane was defined as zero height, i.e. $z=0$.

The scanning area of SWLI was a square box with the size of $834 \mu \mathrm{m}$ $\times 834 \mu \mathrm{m}$, which is large enough to cover a whole RA region on a fracture surface. By positioning the specimen, the scanning box was almost located on the center of an RA region. After scanning, data processing and image reconstructing, the topograph of every RA region was built in terms of the measured height values $(z)$ of the fracture surface with different colors.

For the observations with FIB/SEM and FIB/TEM systems, the samples were prepared in the following steps. (1) A layer of Platinum coating was deposited on the selected location to protect the fracture surface. (2) The profile section within the region was trenched and polished by FIB milling and subsequently imaged by SEM, i.e. FIB-SEM.

Table 1

Fatigue data of the selected specimens A, B, C and D.

\begin{tabular}{llllll}
\hline specimen & $R$ & $\sigma_{\mathrm{a}} / \mathrm{MPa}$ & $\sigma_{\mathrm{m}} / \mathrm{MPa}$ & $N_{\mathrm{f}} /$ cycles & fatigue regime \\
\hline A & -1 & 415 & 0 & $6.63 \times 10^{6}$ & HCF \\
B & -1 & 400 & 0 & $2.84 \times 10^{8}$ & VHCF \\
C & 0.1 & 300 & 367 & $2.24 \times 10^{7}$ & VHCF \\
D & 0.5 & 240 & 720 & $7.82 \times 10^{7}$ & VHCF \\
\hline
\end{tabular}


(3) The TEM sample within the region was cut, lifted, mounted and finally thinned to the dimensions of about $10 \mu \mathrm{m} \times 5 \mu \mathrm{m} \times 50 \mathrm{~nm}$, and then every sample was examined by TEM, i.e. FIB-TEM.

The observations with the tilted and high magnified images for the fractographic features were taken by using FE-SEM with the Zeiss Merlin and a FEI Helios Nanolab 600i. The latter is also a FIB/SEM dual-beam system.

The TEM observation was conducted by using a FEI Talos F200X (acceleration voltage: $200 \mathrm{kV}$ ) and a FEI Tecnai G2 F30 S-Twin (acceleration voltage: $300 \mathrm{kV}$ ). The micro and nano structures underneath the fracture surfaces were analyzed by bright field (BF), dark field (DF) imaging and selected area electron diffraction (SAD) detection with a circular domain of $200 \mathrm{~nm}$ in diameter.

\section{Results and discussion}

\subsection{Topograph of fracture surface}

The SEM and SWLI observations for specimens A, B, C and D are illustrated in Figs. 4-7 with the color bar on the right side of each figure denoting the fracture surface roughness by the height value $z$.

The SEM observations demonstrate that RA and FiE are the typical internal cracking features for the tested titanium alloy with LM and EM in HCF and VHCF regimes, and the RA region is certainly the crack initiation site. As shown in Figs. 4a, 5a and 6a and 7a, the RA and the FiE can be easily identified on the fracture surfaces, which are both pennyshape regions indicated by the two dashed circles. The diameter of the RA regions is between 300 and $600 \mu \mathrm{m}$, and that of FiE is from submillimeter to $1.5 \mathrm{~mm}$.

In addition, a black line $\mathrm{AB}$ on each RA surface of specimens $\mathrm{A}, \mathrm{B}, \mathrm{C}$ and D was drawn as shown in Figs. $4 d, 5 d$ and $6 \mathrm{~d}$ and $7 d$, from which the surface edge curve in relation to each line was observed and then the roughness of each RA region was measured. Thus, the resulted height value $z$ was recorded along the black line from point $A$ to point $B$ in
Figs. 4d, 5d and 6d and 7d, and accordingly plotted in Fig. 8.

\subsubsection{Specimen $A$ under $R=-1$ failed in HCF regime}

Fig. $4 a$ is an SEM image at low magnification showing the whole fracture surface of specimen A. Fig. $4 \mathrm{~b}$ is a topograph showing the whole RA region, where the height range is $34.3 \mu \mathrm{m}$ (from -19.4 to $14.9 \mu \mathrm{m}$ ). Fig. 4c and $d$ are the enlargements of Fig. $4 a$ and b, respectively, showing the morphologies of the RA region.

It is seen from Fig. 4a that the FiE is relatively smooth compared to the RA. The height values of the fracture surface are changed within the RA region or within the SWLI box. As shown in Fig. 4b-d, there are many deep-radial ridges in the center of the RA region and several facet-like appearances with rough surface in the RA center. The facet-like appearances have higher roughness value than those of the facets in EM [18-20] and LM [17,21,25,54] type titanium alloys. Thereby, such facet-like feature is named as "quasi-facet" in this paper.

In fact, this RA center consists of two quasi-facets numbered 01 and 02, and no quasi- or other facets are found away from the RA center. The phenomenon of quasi-facet is a new fractographic feature which is closely related to the crack nucleation under this loading condition of $R$ $=-1$ and failed in HCF regime. The size of quasi-facets 01 and 02 is about $40 \mu \mathrm{m}$ on the projected plane of Fig. 4c. By the comparison between Fig. 4a and c, it is obvious that the RA size is an order of magnitude greater than the quasi-facet size.

The fatigue crack initiation and growth in specimen A can be summarized as follows: (1) quasi-facets 01 and 02 formed independently as two short cracks, (2) they coalesced as a main crack, (3) the main crack outwardly propagated to form an RA and an FiE, and (4) the main crack propagated beyond the FiE leading to final failure.

\subsubsection{Specimen $B$ under $R=-1$ failed in VHCF regime}

Fig. $5 a$ is an SEM image at low magnification showing the whole fracture surface of specimen B. In general, the RA and FiE regions of specimen $B$ are smoother than those of specimen A. It is noted that there
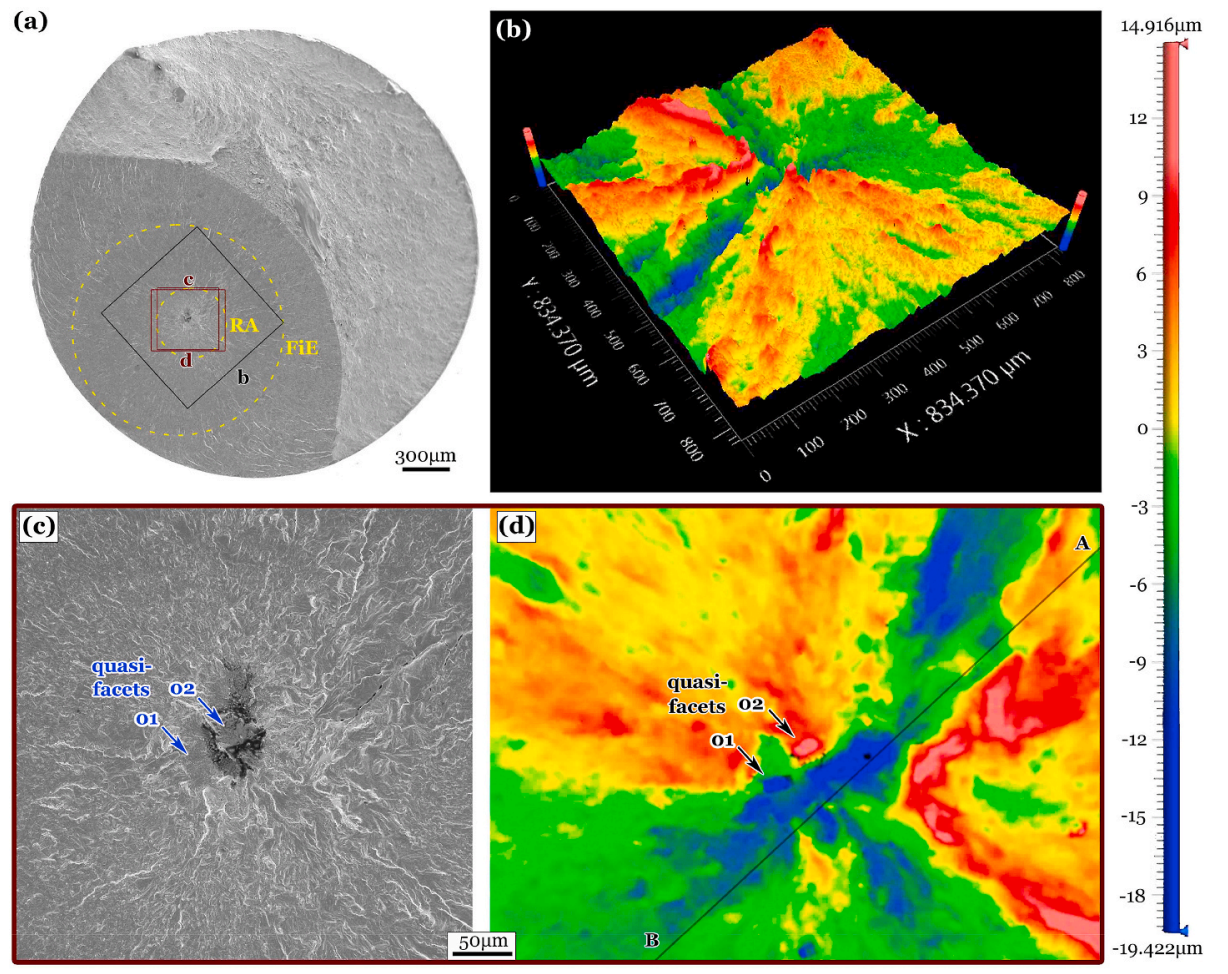

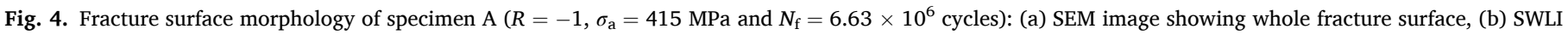

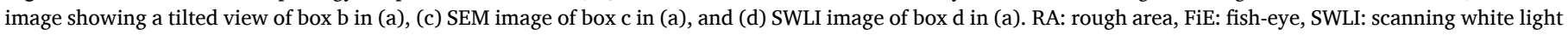
interferometry. 

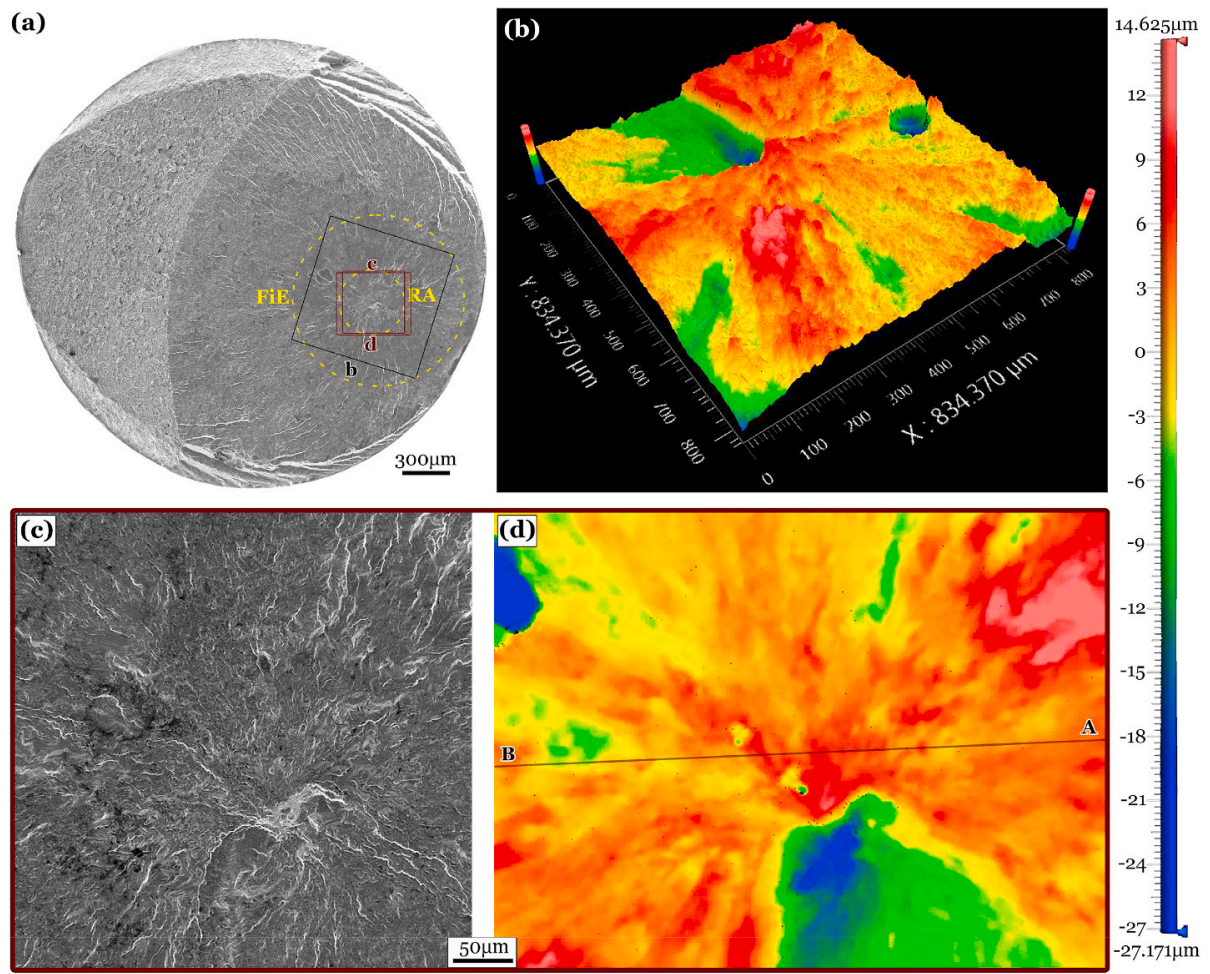

Fig. 5. Fracture surface morphology of specimen B $\left(R=-1, \sigma_{\mathrm{a}}=400 \mathrm{MPa}\right.$ and $N_{\mathrm{f}}=2.84 \times 10^{8}$ cycles): (a) SEM image showing whole fracture surface, (b) SWLI image showing a tilted view of box b in (a), (c) SEM image of box c in (a), and (d) SWLI image of box d in (a). RA: rough area, FiE: fish-eye, SWLI: scanning white light interferometry.
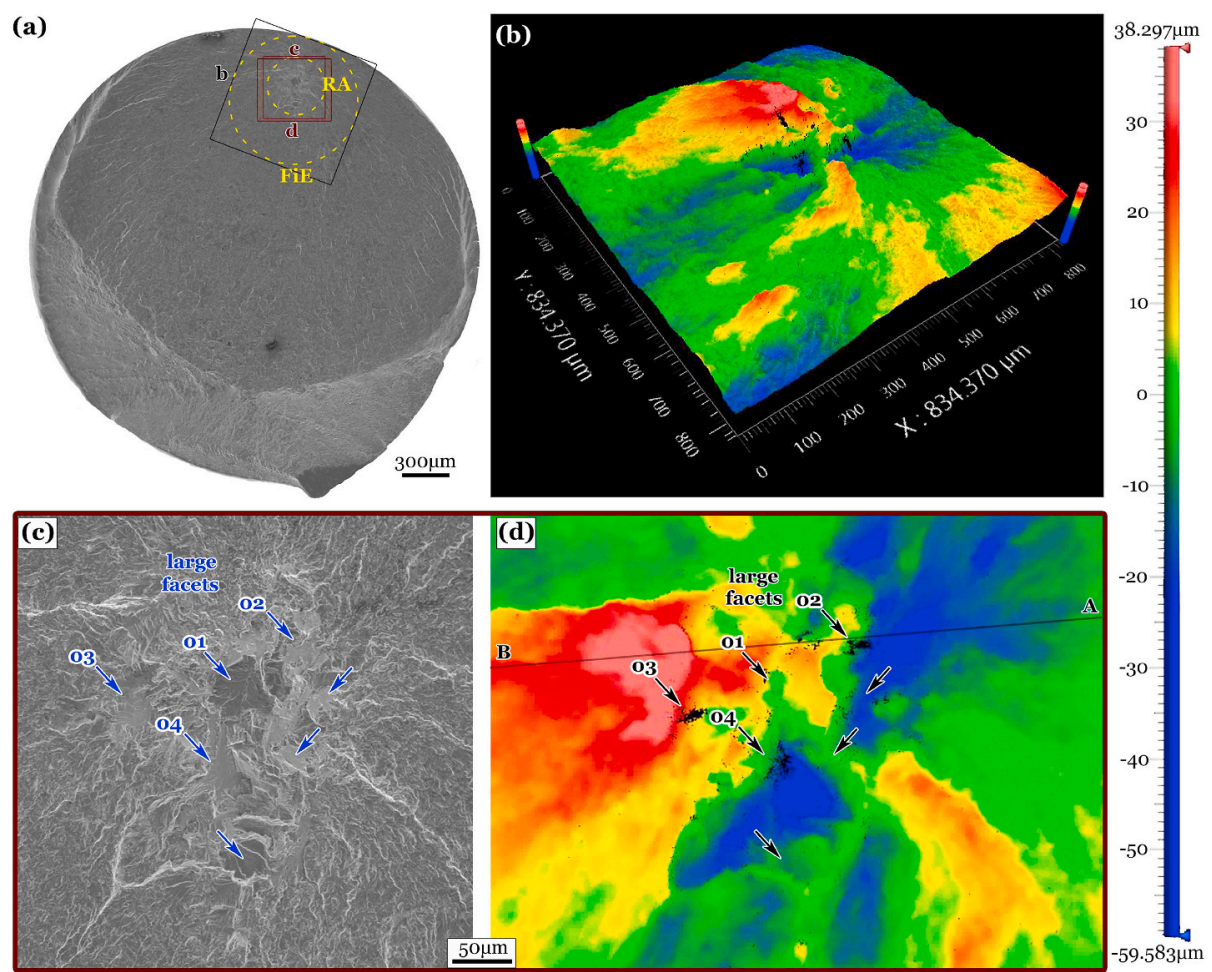

Fig. 6. Fracture surface morphology of specimen $\mathrm{C}\left(R=0.1, \sigma_{\mathrm{a}}=300 \mathrm{MPa}, \sigma_{\mathrm{m}}=367 \mathrm{MPa}, N_{\mathrm{f}}=2.24 \times 10^{7}\right.$ cycles). (a) SEM image showing whole fracture surface, (b) SWLI image showing a tilted view of box b in (a), (c) SEM image of box c in (a), and (d) SWLI image of box d in (a). RA: rough area, FiE: fish-eye, SWLI: scanning white light interferometry. 

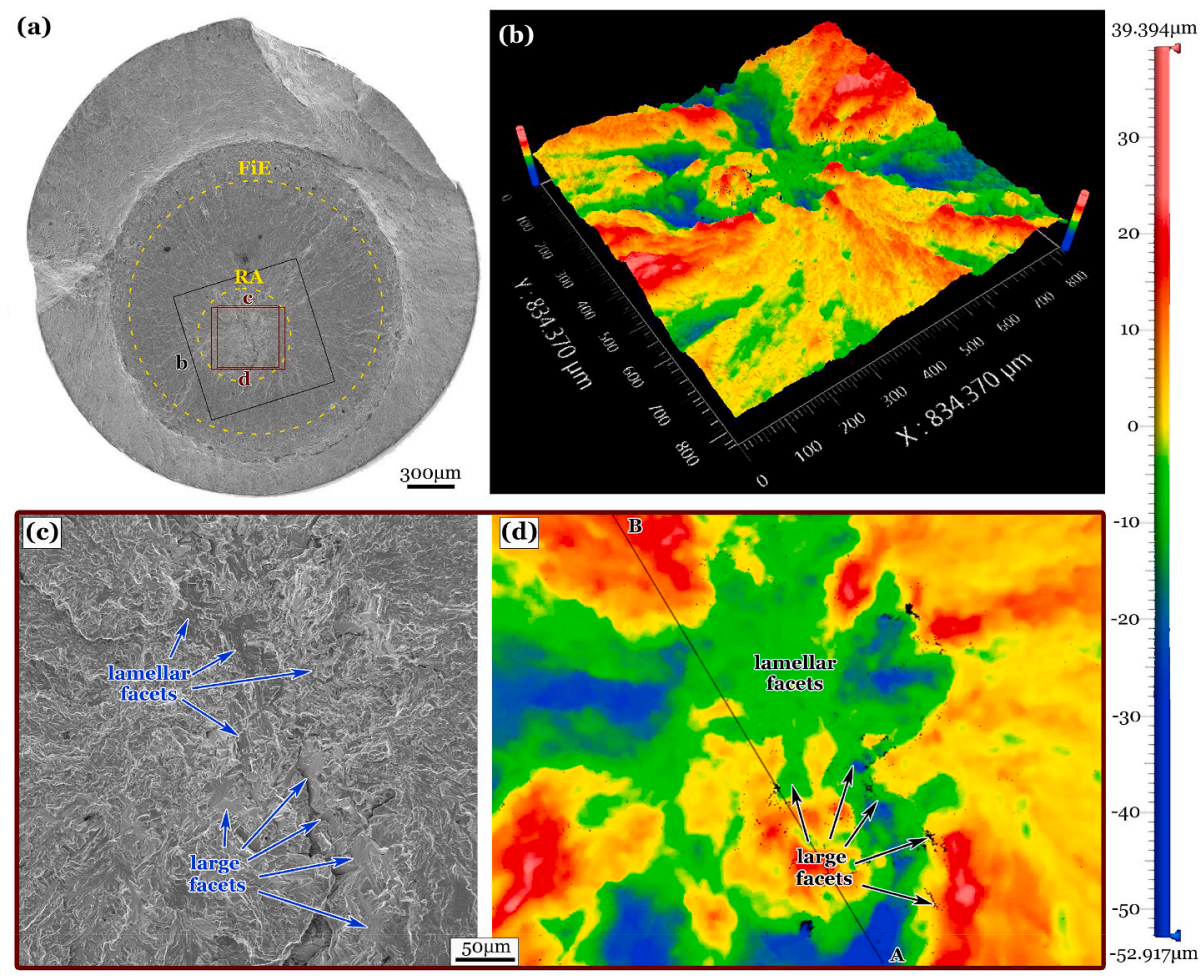

Fig. 7. Fracture surface morphology of specimen $\mathrm{D}\left(R=0.5, \sigma_{\mathrm{a}}=240 \mathrm{MPa}, \sigma_{\mathrm{m}}=720 \mathrm{MPa}\right.$ and $N_{\mathrm{f}}=7.82 \times 10^{7}$ cycles). (a) SEM image showing whole fracture surface, (b) SWLI image showing a tilted view of box b in (a), (c) SEM image of box c in (a), and (d) SWLI image of box d in (a). RA: rough area, FiE: fish-eye, SWLI: scanning white light interferometry.

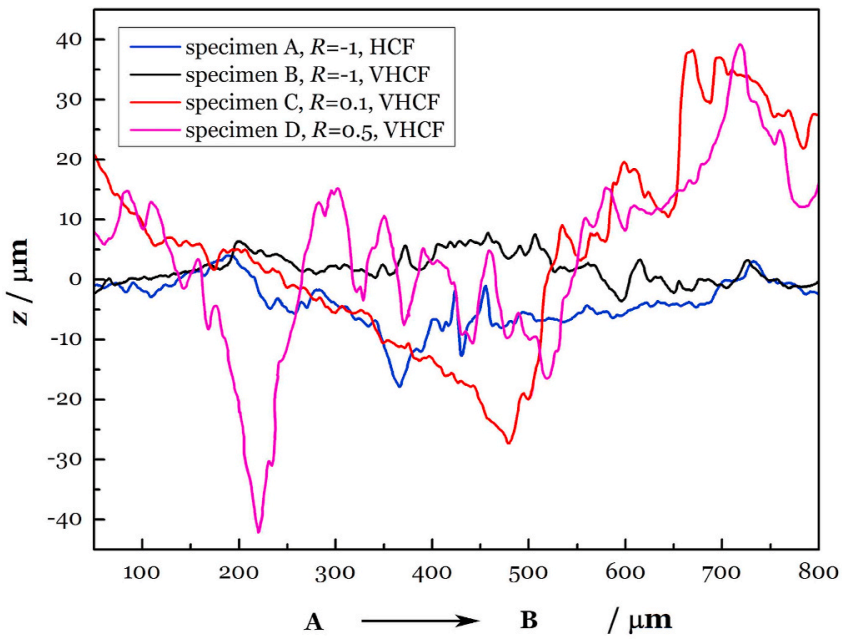

Fig. 8. Fracture surface roughness curves along line $A B$ in specimens $A, B, C$ and $\mathrm{D}$, showing the roughness of the RA regions under different loading conditions.

is a big pit with the size of about $120 \mu \mathrm{m}$ and the depth about $30 \mu \mathrm{m}$ in the FiE region, caused by some metallurgical defect. Fig. $5 \mathrm{~b}$ is the topograph of the RA region and the big pit, where the height range is $41.8 \mu \mathrm{m}$ (from -27.2 to $14.6 \mu \mathrm{m}$ ). By eliminating the datum of the big pit, the height range of RA is about $30 \mu \mathrm{m}$. Fig. $5 \mathrm{c}$ and $\mathrm{d}$ shows the morphologies of the RA region at medium magnification.

As shown in Fig. 5b and d, there are two main crack surface domains of red and blue zones within the RA region. The height range within the red or the blue zone is relatively small and the height difference between the two zones is about $20 \mu \mathrm{m}$. This phenomenon can be explained by the stages of RA formation as follows: (1) the crack of red zone was originated from one site and that of blue zone from another site at a different height level, (2) the initiated cracks propagated and produced the fracture surface of red and blue zones, and (3) the two short cracks coalesced when they well developed. Thus, the resulted fracture surface morphology is caused by the height difference of the two initiated cracks, and stages (1) and (2) of crack initiation consumed the majority of the total fatigue life. Here, line $\mathrm{AB}$ (Fig. $5 \mathrm{~d}$ ) was crossing the red zone for the examination of the fracture surface roughness due to one initiated crack. No facet or other fractographic features were observed in the $\mathrm{RA}$ region for this case.

\subsubsection{Specimen $C$ under $R=0.1$ failed in VHCF regime}

Fig. $6 \mathrm{a}$ is an SEM image at low magnification showing the whole fracture surface of specimen C. Fig. 6b is the topograph of the RA region, where the height range is $97.9 \mu \mathrm{m}$ (from -59.6 to $38.3 \mu \mathrm{m}$ ). The scanning box of SWLI covers the RA and FiE regions and indicates that the roughness of RA is much larger than that of FiE. Fig. $6 \mathrm{c}$ and d shows the RA morphology at medium magnification. The facet morphology is very clear within the RA region, and the size of the large ones is about $40 \mu \mathrm{m}$. They are named as "large facets" and numbered as 01-04. In addition, there are some other facets with relatively small size from a few to 20 $\mu \mathrm{m}$, and the large and small facets are all with equiaxed shape.

The large facet 01 has the largest size among the facets and is possibly the first formed in the crack initiation stage. The fatigue failure process in specimen $\mathrm{C}$ can be summarized as follows: (1) large facet 01 first formed and constituted a short crack, (2) other large facets formed sporadically, (3) large facets merged and became an RA, (4) the RA as a main crack propagated to form a penny shape FiE, and (5) the crack grew beyond the FiE and caused final failure.

The morphology of large facets is a typical fractographic feature of the titanium alloy with both LM and EM for this loading condition of $R$ $=0.1$ and in VHCF regime. Actually, the facet morphology is very common in titanium alloys not only in the tested material, but also in many others with EM [18-20], BM [17,36,39,42-44] and LM [17, 
21-23]. The difference in the present investigation is just in the size and the distribution of the facets. In the titanium alloys with EM or BM, the size of facets is similar to that of equiaxed $\alpha$ grains; and in the LM types, the size of facets is related to that of prior $\beta$ grains.

\subsubsection{Specimen $D$ under $R=0.5$ failed in VHCF regime}

Fig. $7 \mathrm{a}$ is an SEM image at low magnification showing the whole fracture surface of specimen $\mathrm{D}$. The diameter of the RA region is about $600 \mu \mathrm{m}$, which is larger than that of specimens A, B and C. Fig. 7b is the topography of the RA region, where the range of height values is 92.3 $\mu \mathrm{m}$ (from -52.9 to $39.4 \mu \mathrm{m}$ ). In addition, the roughness of FiE in specimen D is also evidently larger than that in specimens A, B and C. Fig. $7 \mathrm{c}$ and $\mathrm{d}$ shows the RA morphology at medium magnification. Many large facets with the size of about $40 \mu \mathrm{m}$, and "lamellar facets" with the length of about $40 \mu \mathrm{m}$ and the width less than $10 \mu \mathrm{m}$ prevail in the RA region. Like in the case of specimen $\mathrm{C}$, there are small facets with similar shape and size distributed in this region. Similarly, the large facet morphology is the typical fractographic feature for this loading condition of $R=0.5$ and failed in VHCF regime.

Thus, the evolution of RA and FiE regions in specimen $\mathrm{D}$ can be described as: (1) a large facet first formed and created a short crack, (2) several other facets successively formed and created other short cracks, (3) initiated short cracks coalesced to form an RA as a main crack with penny shape, and (4) the crack developed beyond the RA to form an FiE that grew and resulted in fatigue failure.

\subsubsection{Comparison of $R A$ roughness for specimens $A, B, C$ and $D$}

The surface edge curves (roughness) along the line AB in Figs. $4 d, 5 d$ and $6 \mathrm{~d}$ and $7 \mathrm{~d}$ are plotted in Fig. 8, indicating the roughness of the RA regions corresponding to the four loading conditions of specimens $\mathrm{A}, \mathrm{B}$, $\mathrm{C}$ and $\mathrm{D}$. Note that line $\mathrm{AB}$ in each fracture surface is a representative of the given specimen and the line passes through different fractographic regions, such as quasi-facet, large facet and/or lamellar facet.

The four curves in Fig. 8 can be classified into two groups: (1) specimens $A$ and $B$, and (2) specimens $C$ and $D$. In group (1) of specimens $\mathrm{A}(R=-1, \mathrm{HCF})$ and $\mathrm{B}(R=-1, \mathrm{VHCF})$, the height value is $-20 \mu \mathrm{m}<z$ $<5 \mu \mathrm{m}$ for the HCF case and $-5 \mu \mathrm{m}<z<10 \mu \mathrm{m}$ for the VHCF case. In group (2) of specimens C ( $R=0.1$, VHCF) and D ( $R=0.5$, VHCF), the height value is $-30 \mu \mathrm{m}<\mathrm{z}<40 \mu \mathrm{m}$ for the case of $R=0.1$ and $-45 \mu \mathrm{m}$ $<\mathrm{z}<40 \mu \mathrm{m}$ for the case of $R=0.5$.

Fig. 8 indicates that the height value is about $20 \mu \mathrm{m}$ for group (1) and about $80 \mu \mathrm{m}$ for group (2). The quadrupled difference in RA roughness implies that there is a different mechanism dominating the crack initiation and early growth in the loading conditions between groups (1) under negative stress ratio and (2) under positive stress ratio, which will be further addressed in the following sections.

\subsection{FIB-SEM and FIB-TEM examination on RA regions}

The topograph of fracture surface indicates that the morphology of RA and FiE prevails in all specimens, quasi-facets only in specimen A, and large facets in specimens $\mathrm{C}$ and $\mathrm{D}$. Then, the microstructure underneath the fracture surfaces was carefully examined with FIB-SEM and FIB-TEM techniques: (1) the RA morphology with quasi-facets was examined in specimen A ( $R=-1, \mathrm{HCF})$, (2) the RA morphology with large facets was examined in specimen C ( $R=0.1$, VHCF), (3) the RA morphology with several facets (large and lamellar) was examined in specimen D ( $R=0.5, \mathrm{VHCF})$, and (4) the RA morphology without specific features was examined in specimen $\mathrm{B}(R=-1$, VHCF $)$.

\subsubsection{Characteristics of $R A$ under $R=-1$ and failed in $H C F$}

Fig. 9a is an SEM image with a tilted angle at medium magnification showing quasi-facets and the neighboring region of specimen A. Quasifacet 01 was selected for the microstructure examination underneath the fracture surface by using FIB-SEM and FIB-TEM. Two profile planes were prepared by FIB within the quasi-facet marked as b and c in Fig. 9a. Fig. $9 \mathrm{~b}$ is an SEM image of profile $b$ and Fig. $9 \mathrm{c}$ is a TEM image of profile c.

Fig. 9b and c shows the wavy edges of the fracture surface at high magnification with only LM in both cross sections of quasi-facet 01 . In Fig. 9c, every lamellar $\alpha$ grain was detected by selected area electron diffraction (SAD). The result of clear isolated spots demonstrates that there is basically no difference of SAD patterns, no matter how the selected area is located, near or away from the fracture surface as shown in Fig. 9d-g. It is proven that all $\alpha$ lamellae have almost the same crystal orientation in the domain. The microstructure characterizations of FIBSEM and FIB-TEM clarify that quasi-facet is formed from an LM domain with similar orientations.

\subsubsection{Characteristics of $R A$ under $R=0.1$ and failed in VHCF regime}

Fig. $10 \mathrm{a}$ is an SEM image with a tilted angle at medium magnification showing the whole RA region of specimen C. The planes of large facets

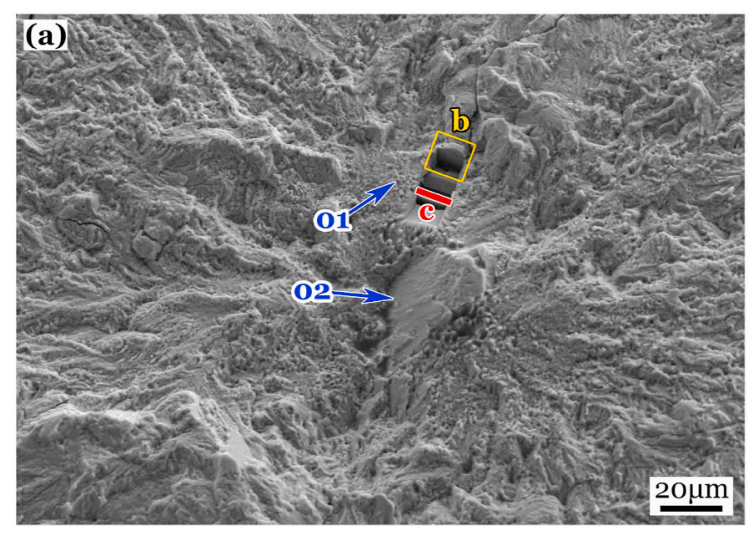

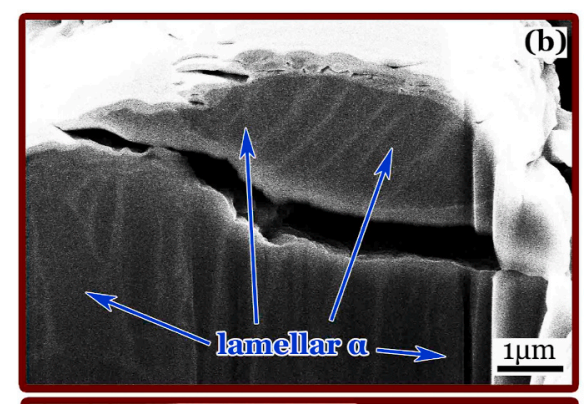

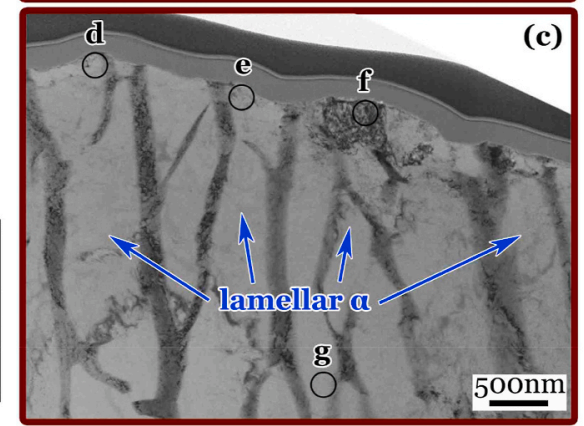

Fig. 9. Fractographic and microstructural features of quasi-facets in the RA region under $R<0$ failed in HCF regime (specimen A): $R=-1, \sigma_{\mathrm{a}}=415 \mathrm{MPa}$, $N_{\mathrm{f}}=6.63 \times 10^{6}$ cycles; (a) SEM image showing a tilted view at the heart of the RA region, (b) FIB-SEM image for section plane $b$ marked by box $b$ in (a), (c) FIB-TEM image for section plane $c$ marked by the red bar c in (a), and ( $-\mathrm{g}$ ) SAD patterns of circular domains $d-g$ in (c). (For interpretation of the references to color in this figure legend, the reader is referred to the Web version of this article.)
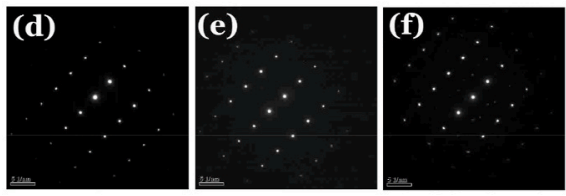

(g)

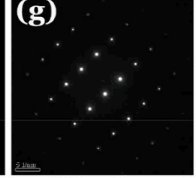



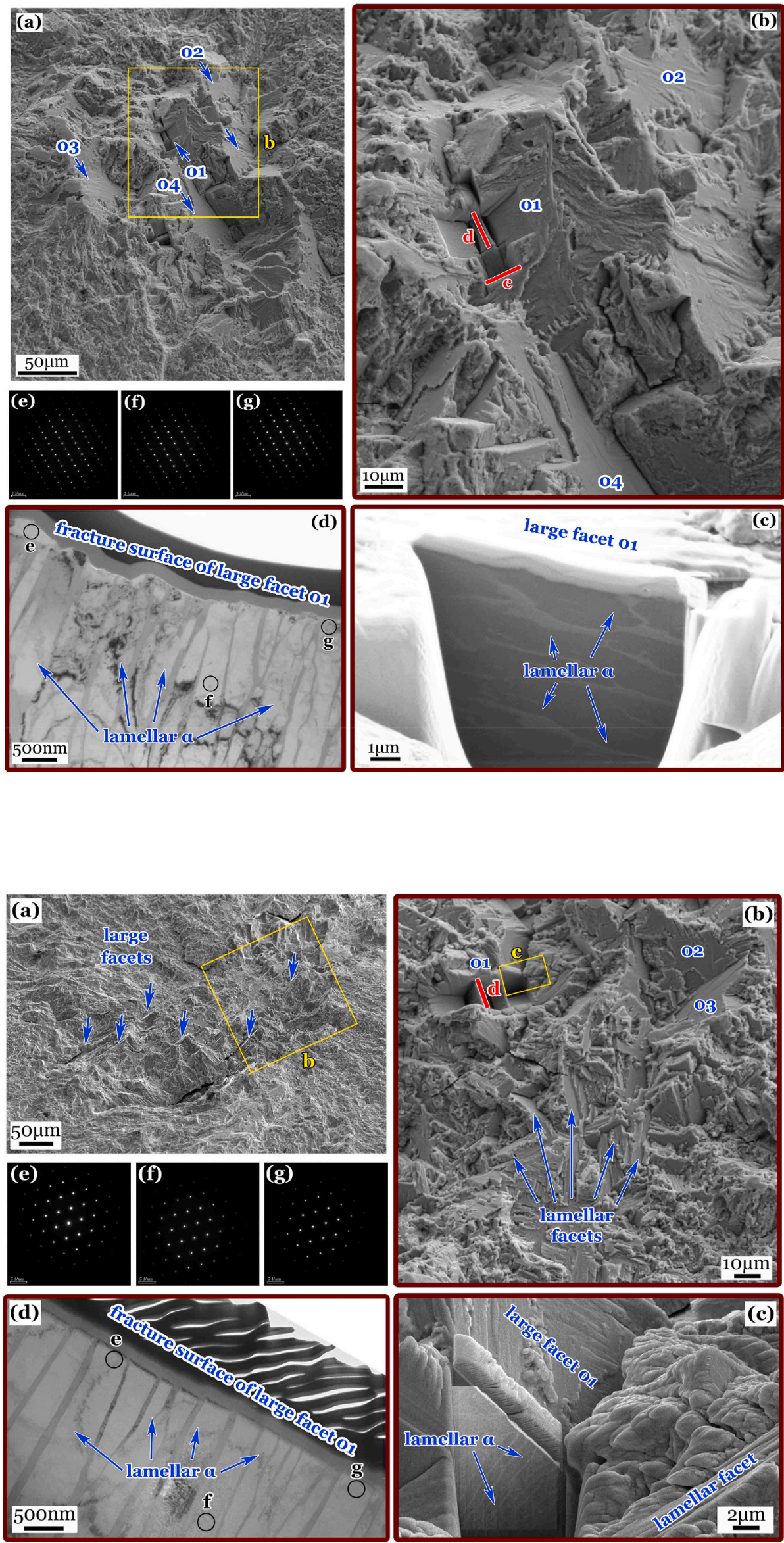

Fig. 10. Fractographic and microstructural features of large facets in the RA region under $R>0$ failed in VHCF regime (specimen C): $R=0.1, \sigma_{\mathrm{a}}=300 \mathrm{MPa}$, $\sigma_{\mathrm{m}}=367 \mathrm{MPa}, N_{\mathrm{f}}=2.24 \times 10^{7}$ cycles; (a) SEM image showing a tilted view of the RA region, (b) SEM image showing the enlargement of box b in (a); (c) FIB-SEM image for section plane c marked by the red bar c in (b); (d) FIB-TEM image for section plane $\mathrm{d}$ marked by the red bar $\mathrm{d}$ in (b); and (e-g) SAD patterns of the circular domains e-g in BF image (d). (For interpretation of the references to color in this figure legend, the reader is referred to the Web version of this article.)
Fig. 11. Fractographic and microstructural features of facets in the RA region under $R>0$ failed in VHCF regime (specimen D): $R=0.5, \sigma_{\mathrm{a}}=240 \mathrm{MPa}, \sigma_{\mathrm{m}}=$ $720 \mathrm{MPa}, N_{\mathrm{f}}=7.82 \times 10^{7}$ cycles; (a) SEM image showing a tilted view of the RA region; (b) SEM image showing detail morphology of box b in (a); (c) FIB-SEM image for profile section c marked by the box $\mathrm{c}$ in (b); (d) FIB-TEM image for profile section $\mathrm{d}$ marked by the red bar $\mathrm{d}$ in (b); and (e-g) SAD patterns of the circular domains e-g in BF image (d). (For interpretation of the references to color in this figure legend, the reader is referred to the Web version of this article.) 
02-04 seem to be parallel each other and large facet 01 has a different orientation. Fig. 10b is an enlarged SEM image with the tilted view to exhibit large facet 01 and the neighboring region. The microstructure underneath large facet 01 was characterized by FIB-SEM and FIB-TEM through two profile samples marked as $\mathrm{c}$ and d in Fig. 10b. Fig. 10c is the SEM image for profile sample c. Fig. $10 \mathrm{~d}$ is the BF image and Fig. 10e-g are the related SAD detections of the profile sample d. Thus, Fig. 10c and d shows the morphologies of LM underneath the fracture surface of large facet 01 , and Fig. 10e-g with almost the same SAD patterns are the typical results from all $\alpha$ lamellae in the sample. This indicates that the colony of LM in Fig. 10d is with similar crystal orientations.

By the comparison of fracture surface of Fig. 10 with Fig. 9, it is seen that the former is smoother than the latter. This is attributed to the different loading conditions between specimens $\mathrm{C}$ and A. Furthermore, as shown in Fig. 10b, large facet 01 is with tree-branch or feather-like morphology, which was also reported in Ref. [54] in a titanium alloy with LM.

\subsubsection{Characteristics of $R A$ under $R=0.5$ and failed in VHCF regime}

Fig. 11a presents an SEM image with a tilted angle at medium magnification of the RA region of specimen $D$, showing a number of large facets with similar orientations as pointed by the arrows. Fig. 11b is a magnified SEM image of a part of Fig. 11a with another tilted angle, showing the morphology of large and lamellar facets. Three large facets in Fig. 11b are labeled as 01-03, and two vertical profile sections within large facet 01 are located (c and d in Fig. 11b). Fig. 11c is an SEM image of location c, showing the morphologies of a lamellar facet and the microstructure in the profile section for large facet 01 . Fig. $11 \mathrm{~d}$ is a TEM BF image of profile d and Fig. 11e-g are the SAD patterns for the domains e-g in Fig. 11d.

It is identified again from the TEM observations that only LM existed in the profile sections within large facet 01 , and all $\alpha$ lamellae are with the same crystal orientation. This phenomenon not only occurs in the quasi-facet in specimen A but also in the large facets in specimens $\mathrm{C}$ and D. It is suggested that there is a similar mechanism of crack initiation from an LM domain with the similar orientation to form a quasi-facet or a large facet. Nevertheless, the roughness of quasi-facet 01 in specimen A is larger than that of the large facets in specimens $C$ and $D$, implying that there is probably a different mechanism of early crack growth in the LM domain.

\subsubsection{Characteristics of $R A$ under $R=-1$ and failed in VHCF regime}

Fig. 12a is an SEM image with a tilted angle at medium magnification showing the morphology of the central area of the RA region for specimen B. Because neither facet nor facet-like patterns appeared in this
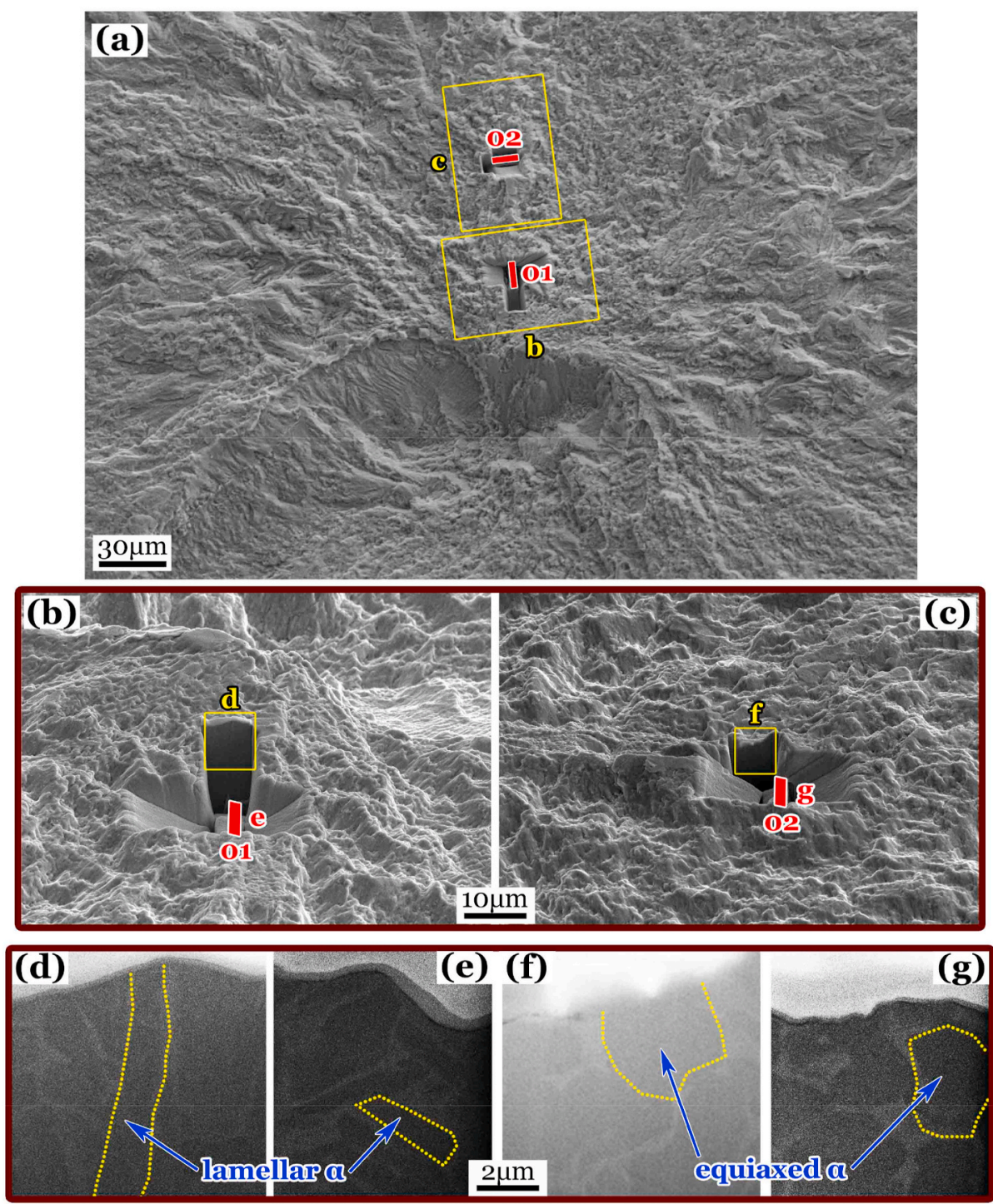

Fig. 12. Fractographic and microstructural features of the RA region under $R<0$ failed in VHCF regime (specimen B): $R=-1, \sigma_{\mathrm{a}}=400 \mathrm{MPa}, N_{\mathrm{f}}=2.84 \times$ $10^{8}$ cycles; (a) SEM image showing a titled view of the RA region, and the red bars 01 and 02 being the locations of TEM samples by FIB; (b, c) high magnified SEM images showing different titled views of boxes $b$ and $c$ in (a); (d) FIB-SEM image for profile section $d$ marked by the box $d$ in (b); (e) FIB-SEM image for profile section marked by number 01 in (b); (f) FIB-SEM image for profile section $\mathrm{f}$ marked by the box $\mathrm{f}$ in (c); and $(\mathrm{g})$ FIB-SEM image for profile section $g$ marked by number 02 in (c). (For interpretation of the references to color in this figure legend, the reader is referred to the Web version of this article.) 
region, two locations (b and $\mathrm{c}$ in Fig. 12a) with no typical features in fracture surface were selected as the representations of the RA region for the detail examination by using FIB-SEM and FIB-TEM. The TEM samples 01 and 02 were cut in the location $b$ and c, respectively, and the plane of sample 02 is perpendicular to that of sample 01 . Then, a profile section perpendicular to the sample plane for each case was carefully polished by FIB technique. Fig. $12 \mathrm{~b}$ and $\mathrm{c}$ are SEM images with a tilted angle at high magnification showing the locations of the TEM samples and the rough morphology of the RA region. The lamellar $\alpha$ grains of the plane $d$ and sample 01 were imaged by SEM and shown in Fig. 12d and e. The equiaxed $\alpha$ grains of another plane $f$ and sample 02 were imaged by SEM and shown in Fig. $12 \mathrm{f}$ and $\mathrm{g}$. The result confirms that the RA region can be formed from either LM or EM of the titanium alloy under the fully reversed cycling $(R=-1)$ up to VHCF regime.

Fig. 13 presents the TEM characterizations for samples 01 and 02 from specimen B (see Fig. 12a). Fig. 13a and b shows the BF images, in which the grain boundaries are clear enough to identify the coarse $\alpha$ grains. Fig. $13 \mathrm{c}$ and $\mathrm{d}$ are the magnified $\mathrm{BF}$ and $\mathrm{DF}$ images for a local region of sample 01, and Fig. 13e and $\mathrm{f}$ are those of sample 02. It is seen from Fig. $13 \mathrm{c}$-f that nanograins prevail just underneath the fracture surface in samples 01 and 02 . Further, nanograins were distributed in a thin layer along the profile of fracture surface. It is obvious that grain size refinement occurred in the RA region of specimen $\mathrm{B}$ under $R=-1$ loading up to VHCF regime. This is the first reported result revealing the detail microstructure morphology of crack initiation region especially showing the nanograin layer underneath the fracture surface of the RA region for the loading case $R=-1$ in VHCF regime on the titanium alloy with both LM and EM.

On the one hand, it is a common result that the formation of nanograins and refined grains was observed in the characteristic regions of crack initiation underneath fracture surfaces for metallic materials under fully reversed axial loading $(R=-1)$ up to VHCF regime such as fine granular area (FGA) in steels [46-49,55], and RA in titanium alloys with BM [15] and with EM [18,19]. On the other hand, no nanograin formation or significant microstructure refinement was observed in quasi-facet of specimen A (HCF, $R=-1$ ) and large facets of specimens $\mathrm{C}$ and D (VHCF, $R>0$ ), similar to the cases in Refs. [15,18,19].

The Numerous Cyclic Pressing (NCP) model $[10,46]$ can well explain this phenomenon, in which the importance of compressive stress and sufficient loading cycles are emphasized. The microstructure evolution underneath the fracture surface is caused by the accumulated plasticity due to the repeated cycling process of crack opening, closing and contacting in the specimen subjected to very high loading cycles. Therefore, the compressive part of applied stress $(R<0)$ and the sufficiently large number of pressing cycles (VHCF) are two necessary conditions for nanograin formation in the crack initiation region.

\subsection{RA formation mechanisms}

From the previous sections 3.1 and 3.2, it is known that the phenomenon of quasi-facets originated from LM domains is a typical fractographic feature in the RA region for specimen A under $R=-1$ experiencing HCF, the phenomenon of large facets originated from LM domains is a typical fractographic feature in the RA region for specimens $\mathrm{C}$ and $\mathrm{D}$ under $R=0.1$ and 0.5 experiencing VHCF, and the phenomenon of nanograins is the typical microstructural feature in the RA region for specimen $\mathrm{B}$ under $R=-1$ experiencing VHCF.

For the sake of further discussion, the loading conditions of specimens A, B, C and D are classified into three categories based on the RA roughness, fractographic features and the NCP concept, i.e. (1) $R>$ 0 failed in VHCF, (2) $R<0$ failed in HCF, and (3) $R<0$ failed in VHCF. There are different mechanisms of crack initiation and early growth for HCF and VHCF failures under $R>0$ and $R<0$ although the specimens all failed by internal cracking failure.

Fig. 14 is a schematic illustration in three categories of internal crack initiation and early growth for the tested titanium alloy with LM and EM loaded in HCF and VHCF regimes. Here, we assume that a crack nucleates from a lamellar $\alpha$ grain and then penetrates the grain along a
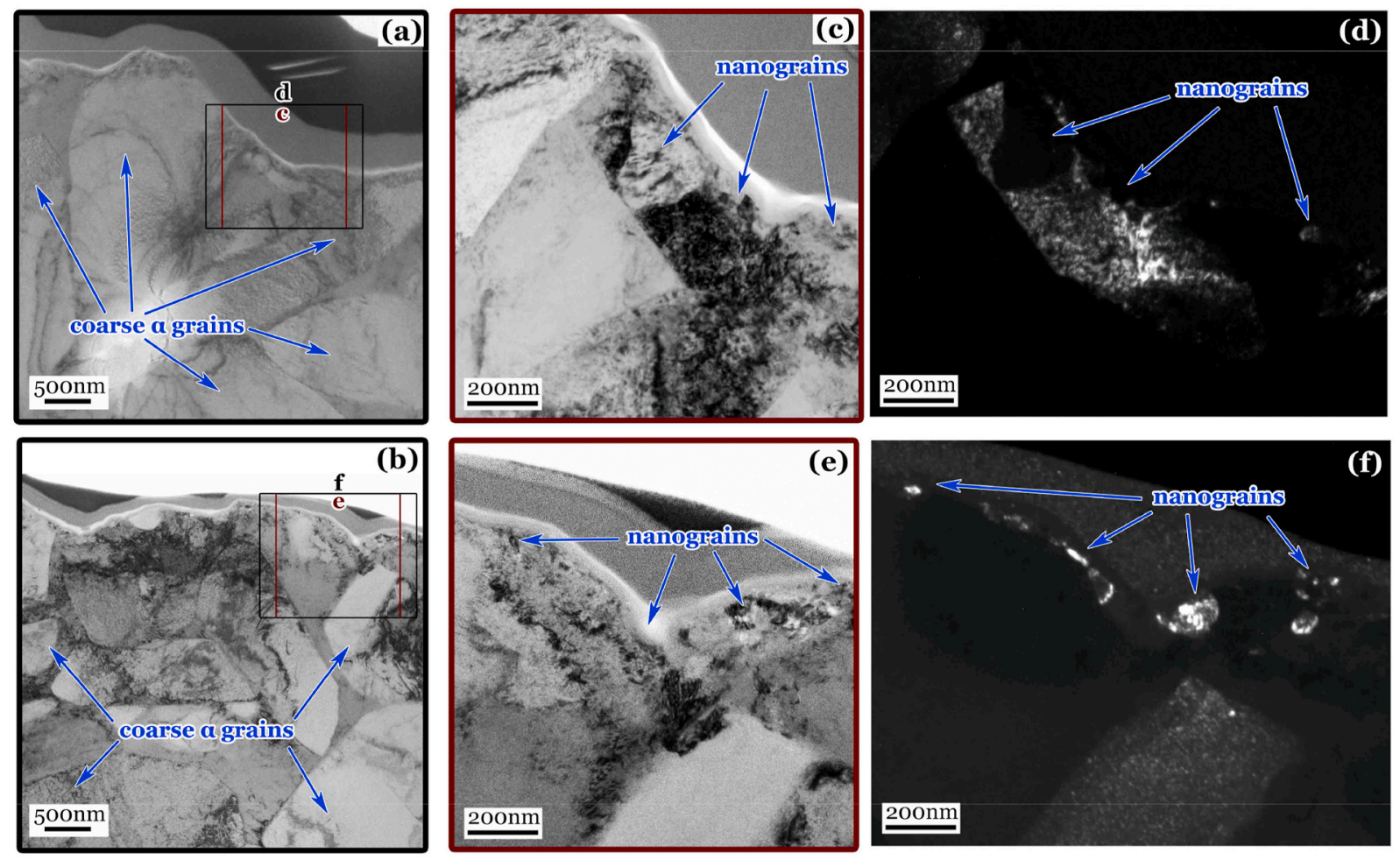

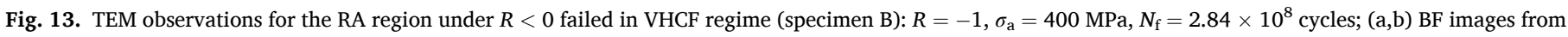

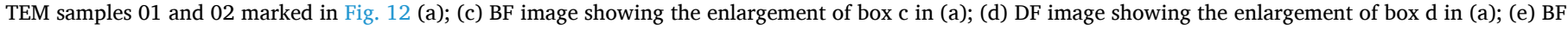
image showing the enlargement of box e in (b); and (f) DF image showing the enlargement of box $f$ in (b). 


\section{internal fatigue-crack initiation and early growth}

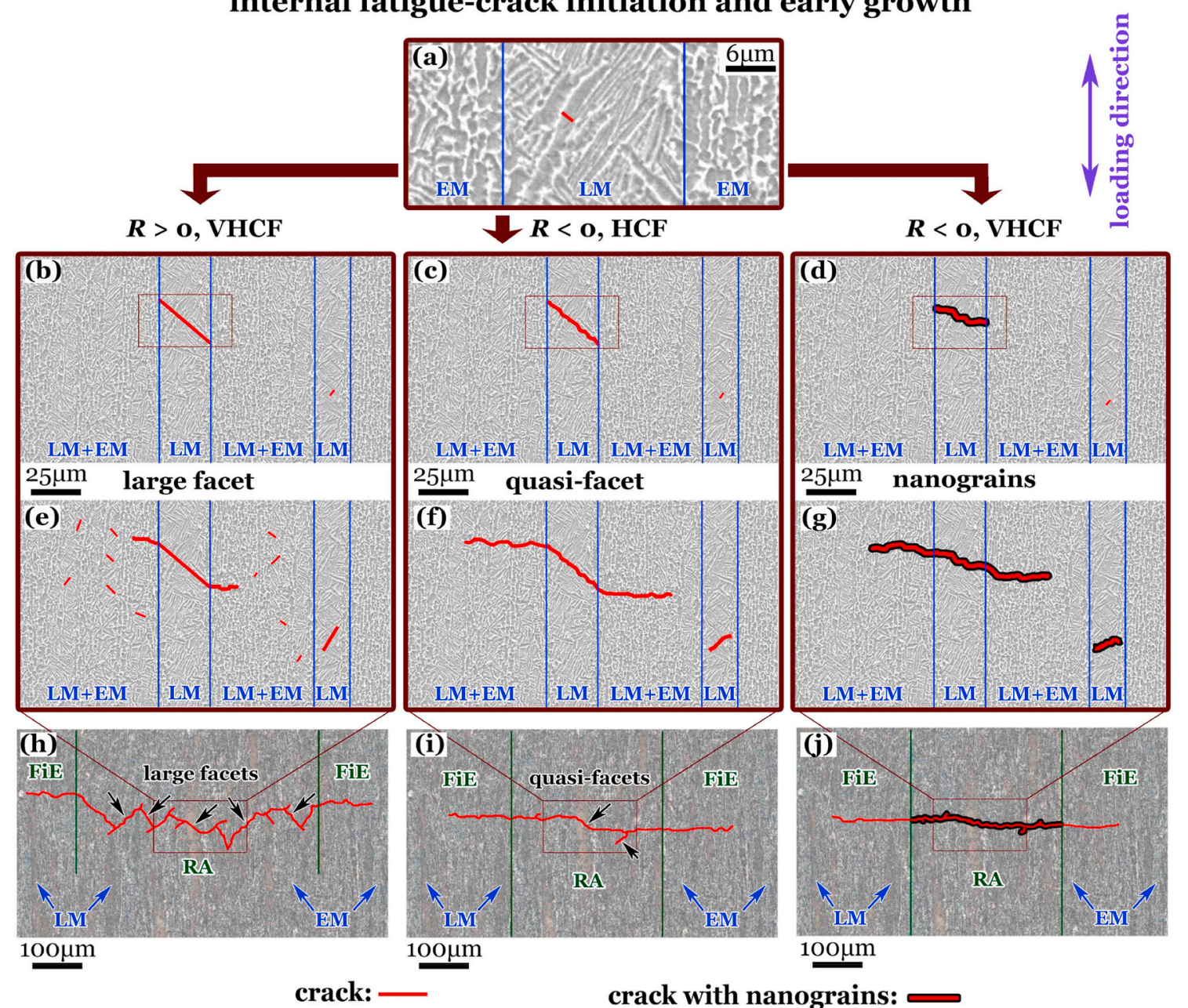

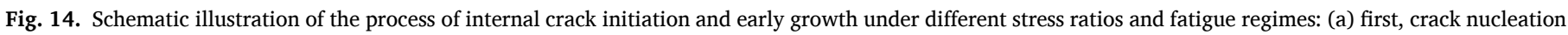

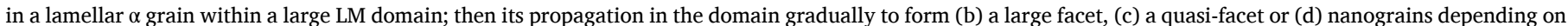

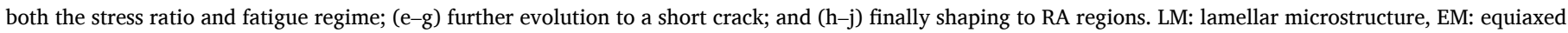
microstructure, RA: rough area, FiE: fish-eye.

specific crystal plane, where the lamella belongs to an LM domain in a large LM column (Fig. 14a). Then, there are three routes of crack evolution under the three loading conditions.

\subsubsection{Mechanism of $R A$ formation in VHCF regime under $R>0$}

Specimens $\mathrm{C}$ and $\mathrm{D}$ were both under $R>0$ and experienced VHCF, with $R$ being 0.1 for specimen $C$ and 0.5 for $\mathrm{D}$. As for the fractographic feature, large facets prevail in the RA region of both specimens. With the increase of $R$, the number of facets increases and the type of facets (large, lamellar or small) is more diverse (referred to the results in sections 3.1.3-3.1.5).

As mentioned in previous sections, a large facet first formed for this case as illustrated in Fig. 14b, which is the short crack initiation stage. This first large facet was resulted from the propagation of the initiated crack over the LM domain with the same orientation on a specific crystal plane, and succeeding cracks initiated at other LM domains (Fig. 14b). Then, the growth of the short crack from the first large facet tended to be perpendicular to the loading direction, and more new facets nucleated in the adjacent regions as schematized in Fig. 14e. After that, as shown in Fig. 14h, the large facets in the LM domains coalesced and the RA formed, subsequently leading to the formation of an FiE with a penny shape to cause the final failure.

It is recalled that the fracture surface of large facet 01 in specimen $D$ at $R=0.5$ is slightly smoother than that in specimen $C$ under $R=0.1$ by the comparison of Fig. 11c and d with Fig. 10c and d. This means that the stress ratio still has an effect on the facet morphology in VHCF regime even when $R>0$, which can be explained by twofold: (1) in the stage of early crack growth, $R$ value will pre-determine the crack path, and (2) after the crack developed, the closure effect due to near field compression will affect the roughness of fracture surface.

\subsubsection{Mechanism of $R A$ formation in HCF regime under $R<0$}

For the case of $R<0$, there is a compressive part of the far field stress, which induces the compressive stress at the near field of initiated crack surfaces to cause crack closure. For specimen A subjected to $R=-1$ and failed in HCF regime, the initiated crack extended to form the first quasifacet with rough surface as shown in Fig. 14c. As in the previous case, some quasi-facet cracks also originated inside the neighboring LM domains with one of them depicted in Fig. 14f. Afterwards, the first quasifacet tended to Mode I crack growth as shown in Fig. 14f. Eventually, a main crack resulted from the first quasi-facet converged with other short cracks emanating from other quasi-facets to form the RA and the FiE in sequence (Fig. 14i).

In the titanium alloy with LM and EM, all quasi- and large facets were originated from large lamellar domains with similar orientations rather than from the equiaxed $\alpha$ grains with small size. This is different from the situation of many other titanium alloys with BM in HCF and VHCF regimes [16,17,36-44]. In those BM types, the facet formation was from 
the equiaxed $\alpha$ grains with larger size than the LM domains with similar orientation. It is suggested that internal crack initiation is prone to occur in the same oriented microstructure domains with large size, such as lamellar microstructure, $\alpha$ grains, or so called "macrozone" of the microtextured regions [23,28,32,33] in HCF and VHCF regimes for titanium alloys.

\subsubsection{Mechanism of $R A$ formation in VHCF regime under $R<0$}

For the specimens failed in VHCF regime under $R<0$, the applied remote stress is lower than that in the case of HCF. As a consequence, the height range of RA region for the former is relatively small and the related fracture surface is without any facet or quasi-facet morphology. It is supposed that the first crack would initiate in an $\alpha$ lamella within an LM domain (Fig. 14a), which could develop to a quasi-facet under a high value of $\sigma_{\mathrm{a}}$ or to a large facet under $R>0$. For the loading case of $R<$ 0 and in VHCF regime, owing to the existence of compressive stress component, the crack propagation did not follow the specific crystallographic orientation, but grew along the path normal to the loading direction. Similar to other cases, cracks also nucleated in the adjacent LM domains (Fig. 14d). Then, the crack continuously grew (Fig. 14g) as a Mode I type to form the RA and the FiE (Fig. 14j).

Simultaneously, the fracture surfaces repeatedly contacted with the crack cyclic opening and closing due to the compressive part of fatigue stress. The microstructure was refined and nanograins were produced at the fracture surfaces when the number of loading cycles was substantially large. In addition, neither nanograins nor refined grains were observed both in quasi-facets of specimen A failed in HCF regime and in large facets of specimens $C$ and D under $R>0$ in VHCF regime. Thus, it is certain that the NCP mechanism $[10,46]$ still dominates the behavior of crack initiation and early growth in VHCF regime for the titanium alloy with both LM and EM.

In the end, it is good to mention that the relation between cracking plane (or direction) and the crystallographic orientation of the microstructure is not investigated in this paper, which is an interesting question that needs further investigation.

\section{Conclusions}

The following main conclusions were drawn for the titanium alloy with lamellar and equiaxed microstructure.

(1) The initiation of internal crack in HCF and VHCF is prone to occur from a large $\alpha$ grain or LM domain with the same crystal orientation in the tested titanium alloy. It is clarified that the size rather than the type (equiaxed or primary $\alpha$ ), the shape, or the distribution of the microstructure domains is the most important factor in being the location of the hot spot for internal crack initiation.

(2) It is first reported that, for the titanium alloy with LM and EM, the rough area (RA) is the characteristic region of crack initiation for VHCF, in which the microstructure refinement and nanograin formation occur in the case of $R=-1$, but in the cases of $R>0$, the microstructure in the crack initiation region remains original coarse feature. This is a new result in support of the proposed Numerous Cyclic Pressing mechanism, in which the contact action between crack surfaces due to the compressive part of applied cyclic stress with sufficient loading cycles is emphasized.

(3) The process of internal crack initiation leading to fatigue failure under different stress ratios is thoroughly revealed. For $R>0$ and failed in VHCF regime, the large LM domains or $\alpha$ grains were cracked to form large, quasi or other type of facets at specific crystal planes. The short cracks initiated at facets coalesced to form the RA region. For $R<0$ and failed in HCF regime, the crack origination was from large LM domains or $\alpha$ grains, and then evolved to the quasi-facets that would converge and propagate to become RA and FiE. For $R<0$ and failed in VHCF regime, the growth of internal cracks originated from large LM domains or $\alpha$ grains is microstructure insensitive without any typical fractographic features such as facets or quasi-facets in the RA region.

\section{CRediT authorship contribution statement}

Xiangnan Pan: Methodology, Investigation, Data curation, Writing original draft. Shouwen Xu: Investigation, Data curation. Guian Qian: Investigation, Validation, Visualization. Alexander Nikitin: Resources, Investigation. Andrey Shanyavskiy: Resources, Visualization. Thierry Palin-Luc: Resources, Visualization. Youshi Hong: Conceptualization, Supervision, Writing - review \& editing.

\section{Declaration of competing interest}

The authors declare that they have no known competing financial interests or personal relationships that could have appeared to influence the work reported in this paper.

\section{Acknowledgments}

Financial supports from the National Natural Science Foundation of China $(11932020,11572325)$ and from the Strategic Priority Research Program of the Chinese Academy of Sciences (XDB22040503, XDB22020201) are greatly acknowledged.

\section{References}

[1] G. Lütjering, J. Williams, Titanium, second ed., Springer-Verlag, Berlin, 2003.

[2] C. Leyens, M. Peters, Titanium and Titanium Alloys, Wiley-VCH Verlag, Weinheim, 2003.

[3] J. Williams, E. Starke, Progress in structural materials for aerospace system, Acta Mater. 51 (2003) 5775-5799.

[4] B. Cowles, High cycle fatigue in aircraft gas turbines - an industry perspective, Int. J. Fract. 80 (1996) 147-163.

[5] S. Suresh, Fatigue of Materials, second ed., Cambridge University Press, Cambridge, 1998.

[6] C. Bathias, P. Paris, Gigacycle Fatigue in Mechanical Practice, Marcel Dekker, New York, 2005.

[7] A. Atrens, W. Hoffelner, T. Duerig, J. Allison, Subsurface crack initiation in high cycle fatigue in Ti-6Al-4V and in a typical martensitic stainless steel, Scripta Metall. 17 (1983) 601-606.

[8] C. Bathias, There is no infinite fatigue life in metallic materials, Fatig. Fract. Eng. Mater. Struct. 22 (1999) 559-565.

[9] M. Zimmermann, Diversity of damage evolution during cyclic loading at very high numbers of cycles, Int. Mater. Rev. 57 (2012) 73-91.

[10] Y. Hong, C. Sun, The nature and the mechanism of crack initiation and early growth for very-high-cycle fatigue of metallic materials - an overview, Theor. Appl. Fract. Mech. 92 (2017) 331-350.

[11] D. Jeddi, T. Palin-Luc, A review about the effects of structural and operational factors on the gigacycle fatigue of steels, Fatig. Fract. Eng. Mater. Struct. 41 (2018) 969-990.

[12] U. Karr, B. Schönbauer, H. Mayer, Near-threshold fatigue crack growth properties of wrought magnesium alloy AZ61 in ambient air, dry air, and vacuum, Fatig. Fract. Eng. Mater. Struct. 41 (2018) 1938-1947.

[13] F. Yoshinaka, T. Nakamura, A. Takeuchi, M. Uesugi, K. Uesugi, Initiation and growth behaviour of small internal fatigue cracks in Ti-6Al-4V via synchrotron radiation microcomputed tomography, Fatig. Fract. Eng. Mater. Struct. 42 (2019) 2093-2105.

[14] Y. Hong, Z. Lei, C. Sun, A. Zhao, Propensities of crack interior initiation and early growth for very-high-cycle fatigue of high strength steels, Int. J. Fatig. 58 (2014) $144-151$.

[15] H. Su, X. Liu, C. Sun, Y. Hong, Nanograin layer formation at crack initiation region for very-high-cycle fatigue of a Ti-6Al-4V alloy, Fatig. Fract. Eng. Mater. Struct. 40 (2017) 979-993.

[16] F. Neal, P. Blenkinsop, Internal fatigue origins in $\alpha-\beta$ titanium alloys, Acta Metall. 24 (1976) 59-63.

[17] R. Nalla, B. Boyce, J. Campbell, J. Peters, R. Ritchie, Influence of microstructure on high-cycle fatigue of Ti-6Al-4V: bimodal vs. lamellar structures, Metall. Mater. Trans. 33 (2002) 899-918.

[18] X. Pan, H. Su, C. Sun, Y. Hong, The behavior of crack initiation and early growth in high-cycle and very-high-cycle fatigue regimes for a titanium alloy, Int. J. Fatig. 115 (2018) 67-78.

[19] X. Pan, Y. Hong, High-cycle and very-high-cycle fatigue behaviour of a titanium alloy with equiaxed microstructure under different mean stresses, Fatig. Fract. Eng. Mater. Struct. 42 (2019) 1950-1964. 
[20] X. Pan, G. Qian, S. Wu, Y. Fu, Y. Hong, Internal crack characteristics in very-highcycle fatigue of a gradient structured titanium alloy, Sci. Rep. 10 (2020) 4742.

[21] D. Eylon, J. Hall, Fatigue behavior of beta processed titanium alloy IMI 685, Metall. Trans. A 8 (1977) 981-990.

[22] J. Zuo, Z. Wang, E. Han, Effect of microstructure on ultra-high cycle fatigue behavior of Ti-6Al-4V, Mater. Sci. Eng.: A-struct. 473 (2008) 147-152.

[23] A. Nikitin, T. Palin-Luc, A. Shanyavskiy, Crack initiation in VHCF regime on forged titanium alloy under tensile and torsion loading modes, Int. J. Fatig. 93 (2016) 318-325.

[24] R.J.H. Wanhill, Consideration of cleavage in alpha titanium, Acta Metall. 21 (1973) 1253-1258.

[25] W.J. Evans, M.R. Bache, Dwell-sensitive fatigue under biaxial loads in the nearalpha titanium alloy IMI685, Int. J. Fatig. 16 (1994) 443-452.

[26] M.R. Bache, Processing titanium alloys for optimum fatigue performance, Int. J. Fatig. 21 (1999) S105-S111.

[27] W.J. Evans, Time dependent effects in fatigue of titanium and nickel alloys, Fatig. Fract. Eng. Mater. Struct. 27 (2004) 543-557.

[28] I. Bantounas, T. Lindley, D. Rugg, D. Dye, Effect of microtexture on fatigue cracking in Ti-6Al-4V, Acta Mater. 55 (2007) 5655-5665.

[29] F. Bridier, P. Villechaise, J. Mendez, Slip and fatigue crack formation processes in an $\alpha / \beta$ titanium alloy in relation to crystallographic texture on different scales, Acta Mater. 56 (2008) 3951-3962.

[30] F.P.E. Dunne, D. Rugg, On the mechanisms of fatigue facet nucleation in titanium alloys, Fatig. Fract. Eng. Mater. Struct. 31 (2008) 949-958.

[31] A.L. Pilchak, A. Bhattacharjee, A.H. Rosenberger, J.C. Williams, Low $\Delta K$ faceted crack growth in titanium alloys, Int. J. Fatig. 31 (2009) 989-994.

[32] I. Bantounas, D. Dye, T. Lindley, The effect of grain orientation on fracture morphology during high-cycle fatigue of Ti-6Al-4V, Acta Mater. 57 (2009) 3584-3595.

[33] I. Bantounas, D. Dye, T. Lindley, The role of microtexture on the faceted fracture morphology in Ti-6Al-4V subjected to high-cycle fatigue, Acta Mater. 58 (2010) 3908-3918.

[34] J. Everaerts, B. verlinden, M. Wevers, Investigation of fatigue crack initiation facets in Ti-6Al-4V using focused ion beam milling and electron backscatter diffraction, J. Microsc. 267 (2017) 57-69.

[35] G. Wu, C. Shi, W. Sha, A. Sha, H. Jiang, Effect of microstructure on the fatigue properties of Ti-6Al-4V titanium alloys, Mater. Des. 46 (2013) 668-674.

[36] X. Liu, C. Sun, Y. Hong, Effects of stress ratio on high-cycle and very-high-cycle fatigue behavior of a Ti-6Al-4V alloy, Mater. Sci. Eng.: A-struct. 622 (2015) 228-235.

[37] K.S. Ravi Chandran, Duality of fatigue failures of materials caused by Poisson defect statistics of competing failure modes, Nat. Mater. 4 (2005) 303-308.

[38] K.S. Ravi Chandran, S. Jha, Duality of the S-N fatigue curve caused by competing failure modes in a titanium alloy and the role of Poisson defect statistics, Acta Mater. 53 (2005) 1867-1881.
[39] X. Liu, C. Sun, Y. Hong, Faceted crack initiation characteristics for high-cycle and very-high-cycle fatigue of a titanium alloy under different stress ratios, Int. J. Fatig. 92 (2016) 434-441.

[40] S. Heinz, F. Balle, G. Wagner, D. Eifler, Analysis of fatigue properties and failure mechanisms of Ti6Al4V in the very high cycle fatigue regime using ultrasonic technology and 3D laser scanning vibrometry, Ultrasonics 53 (2013) 1433-1440.

[41] S. Heinz, D. Eifler, Crack initiation mechanisms of Ti6Al4V in the very high cycle fatigue regime, Int. J. Fatig. 93 (2016) 301-308.

[42] E. Takeuchi, Y. Furuya, N. Nagashima, S. Matsuoka, The effect of frequency on the giga-cycle fatigue properties of a Ti-6Al-4V alloy, Fatig. Fract. Eng. Mater. Struct. 31 (2008) 599-605.

[43] Y. Furuya, E. Takeuchi, Gigacycle fatigue properties of Ti-6Al-4V alloy under tensile mean stress, Mater. Sci. Eng.: A-struct. 598 (2014) 135-140.

[44] W. Li, X. Xing, N. Gao, M. Li, R. Sun, S. Zhou, T. Sakai, Subsurface facets-induced crack nucleation behavior and microstructure-based strength evaluation of titanium alloys in ultra-long life regime, Mater. Sci. Eng.: A-struct. 761 (2019) 138055.

[45] H. Oguma, T. Nakamura, Fatigue crack propagation properties of Ti-6Al-4V in vacuum environments, Int. J. Fatig. 50 (2013) 89-93.

[46] Y. Hong, X. Liu, Z. Lei, C. Sun, The formation mechanism of characteristic region at crack initiation for very-high-cycle fatigue of high-strength steels, Int. J. Fatig. 89 (2016) 108-118.

[47] Q. Jiang, C. Sun, X. Liu, Y. Hong, Very-high-cycle fatigue behavior of a structural steel with and without induced surface defects, Int. J. Fatig. 93 (2016) 352-362.

[48] Y. Hu, C. Sun, Y. Hong, Crack growth rates and microstructure feature of initiation region for very-high-cycle fatigue of a high-strength steel, Fatig. Fract. Eng. Mater. Struct. 41 (2018) 1717-1732.

[49] F. Ritz, C. Stäcker, T. Beck, M. Sander, FGA formation mechanism for X10CrNiMoV12-2-2 and 34CrNiMo6 for constant and variable amplitude tests under the influence of applied mean loads, Fatig. Fract. Eng. Mater. Struct. 41 (2018) 1576-1587.

[50] A. Nikitin, La fatigue gigacyclique d'un alliage de titane, $\mathrm{PhD}$ thesis, Université Paris Nantere, France, 2015 (in English).

[51] A. Nikitin, T. Palin-Luc, A. Shanyavskiy, C. Bathias, Comparison of crack paths in a forged and extruded aeronautical titanium alloy loaded in torsion in the gigacycle fatigue regime, Eng. Fract. Mech. 167 (2016) 259-272.

[52] A. Nikitin, T. Palin-Luc, A. Shanyavskiy, Fatigue crack initiation and growth on an extruded titanium alloy in gigacycle regime: comparison between tension and torsion loadings, Procedia Structural Integrity 2 (2016) 1125-1132.

[53] A. Nikitin, A. Shanyavskiy, N. Beklemishev, Fatigue behavior of titanium alloy under very high cycle fatigue loading, Inorg. Mater.: Applied Research 9 (2018) $75-81$.

[54] D. Eylon, Faceted fracture in beta annealed titanium alloys, Metall. Trans. A 10 (1979) 311-317.

[55] M. Tofique, J. Bergström, K. Svensson, Very high cycle fatigue of cold rolled stainless steels, crack initiation and formation of the fine granular area, Int. J. Fatig. 100 (2017) 238-250. 